Expressions for Form Factors for Inelastic Scattering and Charge Exchange in Plane-Wave, Distorted-Wave, and Coupled-Channels Reaction Formalisms

F. S. Dietrich

September 26, 2006 
This document was prepared as an account of work sponsored by an agency of the United States Government. Neither the United States Government nor the University of California nor any of their employees, makes any warranty, express or implied, or assumes any legal liability or responsibility for the accuracy, completeness, or usefulness of any information, apparatus, product, or process disclosed, or represents that its use would not infringe privately owned rights. Reference herein to any specific commercial product, process, or service by trade name, trademark, manufacturer, or otherwise, does not necessarily constitute or imply its endorsement, recommendation, or favoring by the United States Government or the University of California. The views and opinions of authors expressed herein do not necessarily state or reflect those of the United States Government or the University of California, and shall not be used for advertising or product endorsement purposes.

This work was performed under the auspices of the U.S. Department of Energy by University of California, Lawrence Livermore National Laboratory under Contract W-7405-Eng-48. 


\title{
Expressions for Form Factors for Inelastic Scattering and Charge Exchange in Plane-Wave, Distorted-Wave, and Coupled-Channels Reaction Formalisms
}

\author{
Frank S. Dietrich \\ Lawrence Livermore National Laboratory, Livermore, CA 94550
}

September 25, 2006 


\begin{abstract}
This document is intended to facilitate calculation of inelastic scattering and charge-exchange cross sections in a variety of reaction models, including the plane-wave and distorted-wave approximations and the full coupled-channels treatments. Expressions are given for the coupling potentials between the relevant channels in both coordinate and momentum space. In particular, it is expected that the plane-wave calculations should be useful as a check on the correctness of coupled-channels calculations. The Fourier transform methods used to calculate the plane-wave approximation cross sections are also intended to be used to generate the transition potentials for coupled-channels codes, using a folding model with local effective interactions. Specific expressions are given for calculating transition densities for the folding model in the random phase approximation (RPA).
\end{abstract}




\section{Contents}

1 Introduction $\quad 3$

2 Effective Interactions $\quad 5$

2.1 Central Interaction . . . . . . . . . . . . . . . . . . . . . 5

3 Transition Densities $\quad 8$

3.1 Introduction . . . . . . . . . . . . . . . . . . 8

4 The Distorted-Wave Approximation $\quad 11$

4.1 The DWA Matrix Element . . . . . . . . . . . . . . . . . . . . . 11

4.2 The Transition Potential . . . . . . . . . . . . . . . . . . . . 11

4.3 Cross Section in Plane-Wave Approximation . . . . . . . . . . . . . . . 13

5 Coupled Channels Method $\quad 18$

5.1 Derivation of Radial Coupled Equations . . . . . . . . . . . . . . . . . 18

5.2 Evaluation of Coupling Potentials . . . . . . . . . . . . . . . . . 20

6 Implementation and Computer Codes $\quad 24$

6.1 Coupled-Channels Method . . . . . . . . . . . . . . . . . . . . 24

6.1.1 General Forms for Coupling Potentials . . . . . . . . . . . . . . . 24

6.1.2 Coupling Potentials in Fresco . . . . . . . . . . . . . . . . 26

6.1.3 Coupling Potentials from Tamura . . . . . . . . . . . . . . . 28

6.2 Implementation of Folding Procedure . . . . . . . . . . . . . . . . . . . . . . . . . . . . . . . .

6.2.1 The AllWRLd Code . . . . . . . . . . . . . . . . . . . . 29

6.2.2 The Author's Code, PWA . . . . . . . . . . . . . 29

7 Inelastic Scattering Using RPA Amplitudes 31

7.1 Introduction . . . . . . . . . . . . . . . . . . . . . . 31

7.2 $Z$ Coefficients and Transition Density in the RPA . . . . . . . . . . 33

$\begin{array}{ll}\text { Acknowledgments } & 36\end{array}$

A Angular-Momentum Conventions and Other Definitions 37

A.1 General Remarks . . . . . . . . . . . . . . . . . . . 37

A.2 Definitions of $\bar{Z}$ and $\bar{Z}_{1}$ Coefficients . . . . . . . . . . . . . . . . . 37

A.3 Special Relations among 3-j, 6-j, and 9-j Symbols . . . . . . . . . . . 38 
A.4 Rotation Operators and Matrices . . . . . . . . . . . . . . . . . . . . 39

A.5 Reduced Matrix Elements . . . . . . . . . . . . . . . . . . . . . 41

A.6 B Expressions for Transition Strengths . . . . . . . . . . . . . . . . . 42

B Spectroscopy in Second Quantization 43

B.1 Second Quantization and $Z$ Amplitudes . . . . . . . . . . . . . . 43

B.2 Evaluation of Single-Particle Matrix Elements . . . . . . . . . . . . 46

$\begin{array}{ll}\text { C Fourier-Bessel Expansions } & 48\end{array}$

C.1 Introductory Remarks . . . . . . . . . . . . . . . . . . . . . . 48

C.2 Transformation of Partial-Wave Functions . . . . . . . . . . . . . . . . . 48

C.3 Transformation of Scalar Functions . . . . . . . . . . . . . . . . . 50

C.4 Convolution and Scaling Formulas . . . . . . . . . . . . . . . . . 52

C.5 Delta Function . . . . . . . . . . . . . . . . . . . . . 52

$\begin{array}{ll}\text { Bibliography } & 54\end{array}$ 


\section{Chapter 1}

\section{Introduction}

This report describes the techniques that can be used for the calculation of direct inelastic scattering reactions in nuclei. The application we have in mind is the excitation of highlyexcited states in nuclei by inelastic scattering in the context of so-called "surrogate nuclear reactions." In these reactions, a compound nucleus is formed following the formation of a residual nucleus by a direct reaction, and this compound nucleus subsequently decays according to standard (Hauser-Feshbach) statistical models. An important quantity that must be known for a complete description of the surrogate reaction is the spin-parity distribution of the compound nucleus formed by the direct reaction. This must be investigated separately for each relevant type of direct reaction; that is, inelastic scattering and various stripping and pickup processes. For each type of reaction, the nuclear structure is described by appropriately defined spectroscopic factors. This report focuses on the inelastic scattering reaction.

The basic ingredients in the calculations that are the subject of this report are

- The effective interaction between the projectile and the target nucleons

- The transition density, which contains the nuclear structure information

- Procedures for folding the transition density with the effective interaction to yield a transition potential

- Codes that use the transition density, together with whatever additional input is necessary, to calculate cross sections in the plane-wave, distorted-wave, or coupled-channels formalisms.

Readily available publications and codes for these basic ingredients frequently use conflicting definitions and phase conventions for the needed quantities. This report is intended to ameliorate this situation by presenting a uniform description of the ingredients that can be compared with the existing treatments and can be used to make them work together consistently.

Particular attention is given to momentum-space methods. Plane-wave approximation calculations of cross sections are readily calculated in this formalism, and these provide an important check on the more complicated distorted-wave and coupled-channels methods. Also, momentum-space techniques are useful in calculating the convolutions of interactions 
and transition densities to produce the transition potentials used in the more complicated calculations.

The principal emphasis is on inelastic scattering by spin- 0 or spin- $\frac{1}{2}$ particles. For the latter case, charge exchange is also considered.

The information contained herein is intended as a sufficiently complete description of the ingredients, tools, and techniques that must be employed in specific calculations relevant to the surrogate reaction program. These calculations are under way and will be reported separately. 


\section{Chapter 2}

\section{Effective Interactions}

\subsection{Central Interaction}

We consider local effective interactions between a projectile and a target nucleon and their multipole expansions in coordinate and momentum space. The form of the interaction is restricted by the requirement of rotational invariance and other selection rules. At present we consider only a central interaction with spin-independent and spin-dependent terms, which has the well-known form:

$$
v^{e f f}=v+v^{\sigma} \boldsymbol{\sigma} \cdot \boldsymbol{\sigma}^{\prime}+v^{\tau} \boldsymbol{\tau} \cdot \boldsymbol{\tau}^{\prime}+v^{\sigma \tau} \boldsymbol{\sigma} \cdot \boldsymbol{\sigma}^{\prime} \boldsymbol{\tau} \cdot \boldsymbol{\tau}^{\prime},
$$

where primed quantities refer to the target nucleon, and unprimed to the projectile. The $v$ 's are functions of the distance $\left|\mathbf{r}-\mathbf{r}^{\prime}\right|$, where $\mathbf{r}$ and $\mathbf{r}^{\prime}$ are the positions of the projectile and the target nucleon, respectively. We assume that the interaction may be complex, as well as energy and density dependent. The interaction as shown here assumes that spin and isospin operators can act in the projectile space; i.e. we are considering a spin- $1 / 2$ and isospin- $1 / 2$ projectile such as a nucleon. The appropriate terms should be dropped or modified for other types of projectiles.

We can achieve a more compact notation by defining spin tensors $\mathcal{S}_{S \nu}$, where $S=0$ or 1 ,

$$
\mathcal{S}_{00}=1 \quad \text { and } \quad \mathcal{S}_{1 \nu}=\sigma_{1 \nu},
$$

together with similar quantities $\mathcal{T}_{T q}$ for isospin,

$$
\mathcal{T}_{00}=1 \quad \text { and } \quad \mathcal{T}_{1 q}=\tau_{1 q} .
$$

In the above, $\sigma_{1 \nu}$ and $\tau_{1 q}$ are the spherical components of the vector of Pauli matrices. Using these, the interaction can be written as

$$
v^{e f f}=\sum_{S T} v^{S T}\left(\left|\mathbf{r}-\mathbf{r}^{\prime}\right|\right) \mathcal{S}_{S} \cdot \mathcal{S}_{S}^{\prime} \mathcal{T}_{T} \cdot \mathcal{T}_{T}^{\prime}
$$

A general feature of rotationally invariant interactions is that they may be expressed as a sum of terms, each of which contains a function of the radial coordinates of the two particles, multiplied by a pair of spherical-tensor operators coupled to zero total angular momentum; 
each of these operators is constructed from the angular variables of one of the particles. This will be shown quite easily for the central interactions considered here. For more complicated interactions, such as the spin-orbit and tensor interactions, see other references such as Ref. [1].

The angular-momentum expansions of the first two terms in Eq. 2.1 are

$$
\begin{aligned}
v\left(\left|\mathbf{r}-\mathbf{r}^{\prime}\right|\right) & =\sum_{J M} v_{J}\left(r, r^{\prime}\right) Y_{J M}(\hat{\mathbf{r}}) Y_{J M}^{*}\left(\hat{\mathbf{r}}^{\prime}\right) \\
& =\sum_{J} v_{J}\left(r, r^{\prime}\right) Y_{J}(\hat{\mathbf{r}}) \cdot Y_{J}\left(\hat{\mathbf{r}}^{\prime}\right)
\end{aligned}
$$

for the spin-independent interaction, and

$$
\begin{aligned}
v^{\sigma}(\mid \mathbf{r} & \left.-\mathbf{r}^{\prime} \mid\right) \boldsymbol{\sigma} \cdot \boldsymbol{\sigma}^{\prime} \\
& =\sum_{L \mu} v_{L}^{\sigma}\left(r, r^{\prime}\right) Y_{L \mu}(\hat{\mathbf{r}}) Y_{L \mu}^{*}\left(\hat{\mathbf{r}}^{\prime}\right) \boldsymbol{\sigma} \cdot \boldsymbol{\sigma}^{\prime} \\
& =\sum_{L J M} v_{L}^{\sigma}\left(r, r^{\prime}\right)(-1)^{L+1-J}\left[Y_{L}(\hat{\mathbf{r}}) \times \boldsymbol{\sigma}\right]_{J M}(-1)^{M}\left[Y_{L}\left(\hat{\mathbf{r}}^{\prime}\right) \times \boldsymbol{\sigma}^{\prime}\right]_{J-M} \\
& =\sum_{L J} v_{L}^{\sigma}\left(r, r^{\prime}\right)(-1)^{L+1-J}\left[Y_{L}(\hat{\mathbf{r}}) \times \boldsymbol{\sigma}\right]_{J} \cdot\left[Y_{L}\left(\hat{\mathbf{r}}^{\prime}\right) \times \boldsymbol{\sigma}^{\prime}\right]_{J}
\end{aligned}
$$

for the spin-dependent interaction. The last two expressions for the spin-dependent interaction are gotten from the first by writing out the dot product and then recoupling the $Y_{L}$ and $\boldsymbol{\sigma}$ operators to $J$, separately for each particle. By using the $\mathcal{S}$ and $\mathcal{T}$ tensors, we can combine the above two components and include the isospin operators. The result is

$$
v^{e f f}=\sum_{L S J T} v_{L}^{S T}\left(r, r^{\prime}\right)(-1)^{L+S-J}\left[Y_{L}(\hat{\mathbf{r}}) \times \mathcal{S}_{S}\right]_{J} \cdot\left[Y_{L}\left(\hat{\mathbf{r}}^{\prime}\right) \times \mathcal{S}_{S}^{\prime}\right]_{J} \quad \mathcal{T}_{T} \cdot \mathcal{T}_{T}^{\prime}
$$

Each interaction component contains a factor

$$
v^{S T}=v^{S T}\left(\left|\mathbf{r}-\mathbf{r}^{\prime}\right|\right)
$$

its Fourier transform (see Sec. C.3) is

$$
v^{S T}(q)=\int d \mathbf{r} e^{ \pm i \mathbf{q} \cdot \mathbf{r}} v^{S T}(r)=4 \pi \int_{0}^{\infty} d r r^{2} j_{0}(q r) v^{S T}(r) .
$$

Using the inverse Fourier transform we can express the interaction as

$$
\begin{aligned}
v^{S T}\left(\left|\mathbf{r}-\mathbf{r}^{\prime}\right|\right) & =\frac{1}{(2 \pi)^{3}} \int d \mathbf{q} e^{\mp i \mathbf{q} \cdot\left(\mathbf{r}-\mathbf{r}^{\prime}\right)} v^{S T}(q) \\
& =\frac{1}{(2 \pi)^{3}} \int d \mathbf{q} e^{\mp i \mathbf{q} \cdot \mathbf{r}} e^{ \pm i \mathbf{q} \cdot \mathbf{r}^{\prime}} v^{S T}(q) .
\end{aligned}
$$

We expand each of the exponential factors in the second form above in spherical harmonics according to Eqs. C.3-C.4 and carry out the angular part of the integration. This yields

$$
v^{S T}\left(\left|\mathbf{r}-\mathbf{r}^{\prime}\right|\right)=\sum_{L \mu} \frac{2}{\pi} \int_{0}^{\infty} d q q^{2} j_{L}(q r) j_{L}\left(q r^{\prime}\right) v^{S T}(q) Y_{L \mu}(\hat{\mathbf{r}}) Y_{L \mu}^{*}\left(\hat{\mathbf{r}}^{\prime}\right) .
$$


Since the general form of the spherical harmonic expansion of the part of the interaction depending on $\left|\mathbf{r}-\mathbf{r}^{\prime}\right|$ is

$$
v^{S T}\left(\left|\mathbf{r}-\mathbf{r}^{\prime}\right|\right)=\sum_{L \mu} v_{L}^{S T}\left(r, r^{\prime}\right) Y_{L \mu}(\hat{\mathbf{r}}) Y_{L \mu}^{*}\left(\hat{\mathbf{r}}^{\prime}\right)
$$

we make the identification

$$
v_{L}^{S T}\left(r, r^{\prime}\right)=\frac{2}{\pi} \int_{0}^{\infty} d q q^{2} j_{L}(q r) j_{L}\left(q r^{\prime}\right) v^{S T}(q) .
$$

This expression is useful for the evaluation of the multipole expansion of the interaction in terms of its Fourier transform, which is often more easily determined. 


\section{Chapter 3}

\section{Transition Densities}

\subsection{Introduction}

Several variants of the transition density connecting two states $|i\rangle$ and $|f\rangle$ of a many-body nuclear system are required to describe inelastic scattering and charge exchange reactions. One of them is the matter density evaluated at the position $\mathbf{r}_{t}$,

$$
\rho\left(\mathbf{r}_{t}\right)=\left\langle f\left|\sum_{n} \delta\left(\mathbf{r}_{t}-\mathbf{r}_{n}\right)\right| i\right\rangle
$$

where $\mathbf{r}_{n}$ is regarded as the spatial-coordinate operator of the $n$ 'th particle in the Hilbert space containing the states. Another is the spin density,

$$
\rho_{\nu}^{\sigma}\left(\mathbf{r}_{t}\right)=\left\langle f\left|\sum_{n} \delta\left(\mathbf{r}_{t}-\mathbf{r}_{n}\right) \sigma_{1 \nu}^{n}\right| i\right\rangle
$$

where $\sigma_{1 \nu}^{n}$ is a spherical component of the Pauli matrix $\boldsymbol{\sigma}$ operating on the $n$ 'th nucleon. As is the case with the effective interactions, we combine these definitions and also include isospin by using the spin and isospin tensors $\mathcal{S}_{S \nu}$ and $\mathcal{T}_{T q}$ defined by Eqs. 2.2 and 2.3:

$$
\rho_{S \nu}^{T q}\left(\mathbf{r}_{t}\right)=\left\langle f\left|\sum_{n} \delta\left(\mathbf{r}_{t}-\mathbf{r}_{n}\right) \mathcal{S}_{S \nu}^{n} \mathcal{T}_{T q}^{n}\right| i\right\rangle
$$

We assume that the initial and final states are eigenfunctions of total angular momentum and its projection on an axis, and so we write

$$
\begin{aligned}
|i\rangle & =\left|\alpha_{i} I_{i} M_{i}\right\rangle \\
|f\rangle & =\left|\alpha_{f} I_{f} M_{f}\right\rangle,
\end{aligned}
$$

where $\alpha_{i}$ and $\alpha_{f}$ are additional quantum numbers required to characterize the states. We treat densities that are used with a central interaction only; additional densities relevant to a complete treatment of inelastic scattering, which may involve spin-orbit and tensor interactions, are discussed in Ref. [1]. 
We now make use of angular-momentum algebra to treat the coordinate-space density as

$$
\begin{aligned}
\rho_{S \nu}^{T q}\left(\mathbf{r}_{t}\right)= & \left\langle\alpha_{f} I_{f} M_{f}\left|\sum_{n} \delta\left(\mathbf{r}_{t}-\mathbf{r}_{n}\right) \mathcal{S}_{S \nu}^{n} \mathcal{T}_{T q}^{n}\right| \alpha_{i} I_{i} M_{i}\right\rangle \\
= & \sum_{L \mu}\left\langle\alpha_{f} I_{f} M_{f}\left|\sum_{n} \frac{\delta\left(r_{t}-r_{n}\right)}{r_{t} r_{n}} Y_{L \mu}\left(\hat{\mathbf{r}}_{n}\right) \mathcal{S}_{S \nu}^{n} \mathcal{T}_{T q}^{n}\right| \alpha_{i} I_{i} M_{i}\right\rangle Y_{L \mu}^{*}\left(\hat{\mathbf{r}}_{t}\right) \\
= & \sum_{L \mu J M}(L \mu S \nu \mid J M) \\
& \times\left\langle\alpha_{f} I_{f} M_{f}\left|\sum_{n} \frac{\delta\left(r_{t}-r_{n}\right)}{r_{t} r_{n}}\left[Y_{L}\left(\hat{\mathbf{r}}_{n}\right) \times \mathcal{S}_{S}^{n}\right]_{J M} \mathcal{T}_{T q}^{n}\right| \alpha_{i} I_{i} M_{i}\right\rangle Y_{L \mu}^{*}\left(\hat{\mathbf{r}}_{t}\right) \\
= & \sum_{L \mu J M}(L \mu S \nu \mid J M)\left(I_{i} M_{i} J M \mid I_{f} M_{f}\right)\left(2 I_{f}+1\right)^{-\frac{1}{2}} \\
& \times\left(\alpha_{f} I_{f}\left\|\sum_{n} \frac{\delta\left(r_{t}-r_{n}\right)}{r_{t} r_{n}}\left[Y_{L}\left(\hat{\mathbf{r}}_{n}\right) \times \mathcal{S}_{S}^{n}\right]_{J} \mathcal{T}_{T q}^{n}\right\| \alpha_{i} I_{i}\right) Y_{L \mu}^{*}\left(\hat{\mathbf{r}}_{t}\right) \\
\equiv & 4 \pi \sum_{L \mu J M}(L \mu S \nu \mid J M)\left(I_{i} M_{i} J M \mid I_{f} M_{f}\right) \rho_{L S J}^{T q}\left(r_{t}\right) Y_{L \mu}^{*}\left(\hat{\mathbf{r}}_{t}\right)
\end{aligned}
$$

where we have defined the coordinate-space radial density appearing in the last line as

$$
\rho_{L S J}^{T q}\left(r_{t}\right)=\frac{1}{\sqrt{2 I_{f}+1}}\left(\alpha_{f} I_{f}\left\|\sum_{n} \frac{1}{4 \pi} \frac{\delta\left(r_{t}-r_{n}\right)}{r_{t} r_{n}}\left[Y_{L}\left(\hat{\mathbf{r}}_{n}\right) \times \mathcal{S}_{S}^{n}\right]_{J} \mathcal{T}_{T q}^{n}\right\| \alpha_{i} I_{i}\right) .
$$

We can find similar relations for the momentum-space density by taking the Fourier transform of the above expressions with respect to the variable $\mathbf{r}_{t}$, using Eqs. C.6 and C.7:

$$
\begin{aligned}
\rho_{S \nu}^{T q}(\mathbf{q}) & =\left\langle\alpha_{f} I_{f} M_{f}\left|\sum_{n} e^{ \pm i \mathbf{q} \cdot \mathbf{r}_{n}} \mathcal{S}_{S \nu}^{n} \mathcal{T}_{T q}^{n}\right| \alpha_{i} I_{i} M_{i}\right\rangle \\
& \equiv 4 \pi \sum_{L \mu J M}( \pm i)^{L}(L \mu S \nu \mid J M)\left(I_{i} M_{i} J M \mid I_{f} M_{f}\right) \rho_{L S J}^{T q}(q) Y_{L \mu}^{*}(\hat{\mathbf{q}}),
\end{aligned}
$$

where the momentum-space radial density, found by using Eq. C.50 to transform the radial delta function, is

$$
\rho_{L S J}^{T q}(q)=\frac{1}{\sqrt{2 I_{f}+1}}\left(\alpha_{f} I_{f}\left\|\sum_{n} j_{L}\left(q r_{n}\right)\left[Y_{L}\left(\hat{\mathbf{r}}_{n}\right) \times \mathcal{S}_{S}^{n}\right]_{J} \mathcal{T}_{T q}^{n}\right\| \alpha_{i} I_{i}\right) .
$$

This result is consistent with the definition of Petrovich, Carr, and McManus [1] for the momentum-space matter and spin densities. The factor of $1 / 4 \pi$ was inserted in the definition of the coordinate-space density (Eq. 3.11) to ensure this correspondence.

Next we evaluate the radial densities of Eqs. 3.11 and 3.14, using the second-quantization techniques of Sec. B.1. We insert into Eq. B.8 the the single-particle operator

$$
O_{J}=f_{L}(r)\left[Y_{L}(\hat{\mathbf{r}}) \times \mathcal{S}_{S}\right]_{J}
$$

where

$$
f_{L}(r)=\frac{1}{4 \pi} \frac{\delta\left(r_{t}-r\right)}{r_{t} r} \quad \text { or } \quad j_{L}(q r)
$$


in coordinate or momentum space, respectively. This yields the result

$$
\begin{aligned}
\rho_{L S J}^{T q}\left(r_{t} \text { or } q\right)= & \sum_{\substack{\alpha_{1} j_{1} t_{1} \\
\alpha_{2} j_{2} t_{2}}} \sqrt{\frac{2 I_{i}+1}{2 I_{f}+1}} Z_{t_{2} t_{1}}^{J}\left(\alpha_{2} j_{2}, \alpha_{1} j_{1}\right) \\
& \quad \times\left(\alpha_{2} j_{2} t_{2}\left\|f_{L}(r)\left[Y_{L}(\hat{\mathbf{r}}) \times \mathcal{S}_{S}\right]_{J}\right\| \alpha_{1} j_{1} t_{1}\right) \mathcal{T}_{T q}^{t_{2} t_{1}},
\end{aligned}
$$

where the isospin matrix element is $\mathcal{T}_{T q}^{t_{2} t_{1}}=\left\langle\frac{1}{2} t_{2}\left|\mathcal{T}_{T q}\right| \frac{1}{2} t_{1}\right\rangle$; see Table B.1 for numerical values. In this expression the density is a sum over reduced matrix elements between single-particle states weighted by spectroscopic amplitudes $Z_{t_{2} t_{1}}^{J}\left(\alpha_{2} j_{2}, \alpha_{1} j_{1}\right)$, which are defined in Sec. B.1. Explicit formulas for evaluation of the reduced matrix elements are given in Sec. B.2.

For inelastic scattering we can define neutron and proton densities corresponding to the parts of the above equation with $t_{1}=t_{2}=\frac{1}{2}$ and $t_{1}=t_{2}=-\frac{1}{2}$, respectively. Specifically, these densities are defined by

$$
\begin{aligned}
& \rho_{L S J}^{00} \equiv \mathcal{T}_{00}^{\frac{1}{2} \frac{1}{2}} \rho_{L S J}^{n}+\mathcal{T}_{00}^{-\frac{1}{2}-\frac{1}{2}} \rho_{L S J}^{p}=\rho_{L S J}^{n}+\rho_{L S J}^{p} \\
& \rho_{L S J}^{10} \equiv \mathcal{T}_{10}^{\frac{1}{2} \frac{1}{2}} \rho_{L S J}^{n}+\mathcal{T}_{10}^{-\frac{1}{2}-\frac{1}{2}} \rho_{L S J}^{p}=\rho_{L S J}^{n}-\rho_{L S J}^{p}
\end{aligned}
$$

Similarly, densities used in charge exchange reactions can be defined as

$$
\begin{aligned}
& \rho_{L S J}^{11} \equiv \mathcal{T}_{11}^{\frac{1}{2}-\frac{1}{2}} \rho_{L S J}^{n \leftarrow p}=-\sqrt{2} \rho_{L S J}^{n \leftarrow p} \\
& \rho_{L S J}^{1-1} \equiv \mathcal{T}_{1-1}^{-\frac{1}{2} \frac{1}{2}} \rho_{L S J}^{p \leftarrow n}=\sqrt{2} \rho_{L S J}^{p \leftarrow n} .
\end{aligned}
$$

The same equations 3.18-3.21 can be used for definitions of neutron, proton, and chargeexchange densities based on $\rho_{S \nu}^{T q}\left(\mathbf{r}_{t}\right.$ or $\left.\mathbf{q}\right)$. 


\section{Chapter 4}

\section{The Distorted-Wave Approximation}

\subsection{The DWA Matrix Element}

We consider inelastic scattering of a single nucleon from one orbital to another outside a spinzero core. This restriction will be removed later my using a second-quantized description of the target. We also consider only the simplest type of interaction, a local, spin-independent interaction between the projectile and the nucleons of the target. Under these conditions we can write the $T$-matrix element as

$$
T_{\mathbf{k}^{\prime} \mathbf{k}}=\int d \mathbf{r} \tilde{\chi}_{\mathbf{k}^{\prime}}^{(-) *}(\mathbf{r}) U(\mathbf{r}) \chi_{\mathbf{k}}^{(+)}(\mathbf{r}),
$$

where we have suppressed possible projectile quantum numbers other than the wave number; $\mathbf{r}$ is the positional coordinate of the projectile. The initial and final wave functions are distorted waves with appropriate boundary conditions and with proper attention to biorthogonality if the distorting potential is complex. The continuum wave functions are box-normalized within a volume $\Omega$.

\subsection{The Transition Potential}

The transition potential $U(\mathbf{r})$ is

$$
\begin{aligned}
U(\mathbf{r}) & =\left\langle\alpha_{f} I_{f} M_{f}\left|\sum_{n} \sum_{S T} v^{S T}\left(\left|\mathbf{r}-\mathbf{r}_{n}\right|\right) \mathcal{S}_{S} \cdot \mathcal{S}_{S}^{n} \mathcal{T}_{T} \cdot \mathcal{T}_{T}^{n}\right| \alpha_{i} I_{i} M_{i}\right\rangle \\
& =\sum_{S \nu T q} \mathcal{S}_{S \nu}^{\dagger} \mathcal{T}_{T q}^{\dagger} \int d \mathbf{r}_{t} v^{S T}\left(\left|\mathbf{r}-\mathbf{r}_{t}\right|\right)\left\langle\alpha_{f} I_{f} M_{f}\left|\sum_{n} \delta\left(\mathbf{r}_{t}-\mathbf{r}_{n}\right) \mathcal{S}_{S \nu}^{n} \mathcal{T}_{T q}^{n}\right| \alpha_{i} I_{i} M_{i}\right\rangle \\
& =\sum_{S \nu T q} \mathcal{S}_{S \nu}^{\dagger} \mathcal{T}_{T q}^{\dagger} \int d \mathbf{r}_{t} v^{S T}\left(\left|\mathbf{r}-\mathbf{r}_{t}\right|\right) \rho_{S \nu}^{T q}\left(\mathbf{r}_{t}\right) \\
& \equiv \sum_{S \nu T q} \mathcal{S}_{S \nu}^{\dagger} \mathcal{T}_{T q}^{\dagger} U_{S \nu}^{T q}(\mathbf{r})
\end{aligned}
$$

where we have inserted the expression of Eq. 2.4 for the effective interaction between the projectile and the $n$ 'th target nucleon. The initial and final states of the target are described by $\left|\alpha_{i} I_{i} M_{i}\right\rangle$ and $\left|\alpha_{f} I_{f} M_{f}\right\rangle$, respectively; the angular momentum and m-projection 
are shown explicitly, and additional quantum numbers are represented by $\alpha_{f}$ and $\alpha_{i}$. The spin and isospin operators in the projectile space in the second through fourth lines are $\mathcal{S}_{S \nu}^{\dagger}=(-1)^{\nu} \mathcal{S}_{S-\nu}$ and $\mathcal{T}_{T q}^{\dagger}=(-1)^{q} \mathcal{T}_{T-q}$. In the third line we have use the definition of the transition density from Eq. 3.6, and in the last line we have defined a transition potential depending only on the spatial coordinate of the projectile,

$$
U_{S \nu}^{T q}(\mathbf{r})=\int d \mathbf{r}_{t} v^{S T}\left(\left|\mathbf{r}-\mathbf{r}_{t}\right|\right) \rho_{S \nu}^{T q}\left(\mathbf{r}_{t}\right)
$$

We insert the angular momentum expansions of Eqs. 2.16 and 3.10 for the interaction and density factors in the above expression. After carrying out the angular integral we get

$$
U_{S \nu}^{T q}(\mathbf{r})=4 \pi \sum_{L \mu J M}(L \mu S \nu \mid J M)\left(I_{i} M_{i} J M \mid I_{f} M_{f}\right) \int_{0}^{\infty} d r_{t} r_{t}^{2} v_{L}^{S T}\left(r, r_{t}\right) \rho_{L S J}^{T q}\left(r_{t}\right) Y_{L \mu}^{*}(\hat{\mathbf{r}})
$$

We define a radial transition potential $U_{L S J}^{T q}(r)$ by

$$
U_{S \nu}^{T q}(\mathbf{r})=\sum_{L \mu J M}(L \mu S \nu \mid J M)\left(I_{i} M_{i} J M \mid I_{f} M_{f}\right) U_{L S J}^{T q}(r) Y_{L \mu}^{*}(\hat{\mathbf{r}})
$$

where

$$
U_{L S J}^{T q}(r)=4 \pi \int_{0}^{\infty} d r_{t} r_{t}^{2} v_{L}^{S T}\left(r, r_{t}\right) \rho_{L S J}^{T q}\left(r_{t}\right)
$$

The coordinate-space radial density $\rho_{L S J}^{T q}\left(r_{t}\right)$ is defined in Eq. 3.11. An alternative expression for the transition potential is gotten by inserting the momentum-space expression for $v_{L}^{S T}\left(r, r_{t}\right)$ from Eq. 2.17 into the last expression, which results in

$$
U_{L S J}^{T q}(r)=\frac{2}{\pi} \int_{0}^{\infty} d q q^{2} j_{L}(q r) v^{S T}(q) \rho_{L S J}^{T q}(q)
$$

where the momentum-space radial density is defined in Eq. 3.14 and is obtained from the coordinate-space radial density by the Fourier transformation of Eq. C.7.

Eq. 4.4 expresses the transition potential as the convolution of an interaction with a density function, and therefore the Fourier transform of the potential can be represented as the product of the Fourier transforms of its components. Thus the transition potential in momentum space is

$$
\begin{aligned}
U(\mathbf{q}) & =\sum_{S \nu T q} \mathcal{S}_{S \nu}^{\dagger} \mathcal{T}_{T q}^{\dagger} U_{S \nu}^{T q}(\mathbf{q}), \quad \text { with } \\
U_{S \nu}^{T q}(\mathbf{q}) & =v^{S T}(q) \rho_{S \nu}^{T q}(\mathbf{q}) .
\end{aligned}
$$

The momentum-space transition density in the last equation is given by Eq. 3.12, and we multiply it by $v^{S T}(q)$ to get

$$
U_{S \nu}^{T q}(\mathbf{q})=4 \pi \sum_{L \mu J M}( \pm i)^{L}(L \mu S \nu \mid J M)\left(I_{i} M_{i} J M \mid I_{f} M_{f}\right) v^{S T}(q) \rho_{L S J}^{T q}(q) Y_{L \mu}^{*}(\hat{\mathbf{q}})
$$


We define a radial transition potential in momentum space $U_{L S J}^{T q}(q)$ by

$$
U_{S \nu}^{T q}(\mathbf{q})=\sum_{L \mu J M}( \pm i)^{L}(L \mu S \nu \mid J M)\left(I_{i} M_{i} J M \mid I_{f} M_{f}\right) U_{L S J}^{T q}(q) Y_{L \mu}^{*}(\hat{\mathbf{q}})
$$

where

$$
U_{L S J}^{T q}(q)=4 \pi v^{S T}(q) \rho_{L S J}^{T q}(q)
$$

\subsection{Cross Section in Plane-Wave Approximation}

We treat the projectile as having spin and isospin $\frac{1}{2}$. In this case the plane-wave expressions for the initial and final states are

$$
\chi_{\mathbf{k} \sigma t}^{(+)}(\mathbf{r})=\frac{1}{\sqrt{\Omega}} e^{i \mathbf{k} \cdot \mathbf{r}} \chi_{\frac{1}{2} \sigma} \chi_{\frac{1}{2} t}^{i s o} \quad \text { and } \quad \tilde{\chi}_{\mathbf{k}^{\prime} \sigma^{\prime} t^{\prime}}^{(-)}(\mathbf{r})=\frac{1}{\sqrt{\Omega}} e^{i \mathbf{k}^{\prime} \cdot \mathbf{r}} \chi_{\frac{1}{2} \sigma^{\prime}} \chi_{\frac{1}{2} t^{\prime}}^{i s o}
$$

and we see that the $T$-matrix element is

$$
\begin{aligned}
T_{\mathbf{k}^{\prime} \sigma^{\prime}, \mathbf{k} \sigma}^{t^{\prime}} & =\frac{1}{\Omega} \int d \mathbf{r} e^{-i \mathbf{k}^{\prime} \cdot \mathbf{r}}\left\langle\frac{1}{2} \sigma^{\prime}, \frac{1}{2} t^{\prime}|U(\mathbf{r})| \frac{1}{2} \sigma, \frac{1}{2} t\right\rangle e^{i \mathbf{k} \cdot \mathbf{r}} \\
& =\frac{1}{\Omega} \int d \mathbf{r} e^{ \pm i \mathbf{q} \cdot \mathbf{r}}\left\langle\frac{1}{2} \sigma^{\prime}, \frac{1}{2} t^{\prime}|U(\mathbf{r})| \frac{1}{2} \sigma, \frac{1}{2} t\right\rangle \\
& =\frac{1}{\Omega}\left\langle\frac{1}{2} \sigma^{\prime}, \frac{1}{2} t^{\prime}|U(\mathbf{q})| \frac{1}{2} \sigma, \frac{1}{2} t\right\rangle,
\end{aligned}
$$

where in the second expression above the upper sign is chosen if $\mathbf{q}$ is defined as $\mathbf{k}-\mathbf{k}^{\prime}$, and the lower sign if it is defined as $\mathbf{k}^{\prime}-\mathbf{k}$. By using the expression Eq. 4.11 for the transition potential the $T$-matrix element becomes

$$
T_{\mathbf{k}^{\prime} \sigma^{\prime}, \mathbf{k} \sigma}^{t^{\prime} t}=\frac{1}{\Omega} \sum_{S \nu T q}\left\langle\frac{1}{2} \sigma^{\prime}\left|(-1)^{\nu} \mathcal{S}_{S-\nu}\right| \frac{1}{2} \sigma\right\rangle\left\langle\frac{1}{2} t^{\prime}\left|(-1)^{q} \mathcal{T}_{T-q}\right| \frac{1}{2} t\right\rangle U_{S \nu}^{T q}(\mathbf{q}) .
$$

We use the Wigner-Eckart theorem to evaluate the spin matrix element as

$$
\begin{aligned}
\left\langle\frac{1}{2} \sigma^{\prime}\left|(-1)^{\nu} \mathcal{S}_{S-\nu}\right| \frac{1}{2} \sigma\right\rangle & =\frac{1}{\sqrt{2}}(-1)^{\nu}\left(\frac{1}{2} \sigma S-\nu \mid \frac{1}{2} \sigma^{\prime}\right)\left(\frac{1}{2}|| \mathcal{S}_{S} \| \frac{1}{2}\right) \\
& =(-1)^{\nu} \sqrt{2 S+1}\left(\frac{1}{2} \sigma S-\nu \mid \frac{1}{2} \sigma^{\prime}\right) \\
& =\sqrt{2}(-1)^{\frac{1}{2}-\sigma^{\prime}}\left(\frac{1}{2} \sigma^{\prime} \frac{1}{2}-\sigma \mid S-\nu\right),
\end{aligned}
$$

where we use the value $\left(\frac{1}{2}\left\|\mathcal{S}_{S}\right\| \frac{1}{2}\right)=\sqrt{2(2 S+1)}$ in the second line and note that $\nu=\sigma-\sigma^{\prime}$ in the third. We can therefore rewrite the T-matrix of Eq. 4.20 as

$$
T_{\mathbf{k}^{\prime} \sigma^{\prime}, \mathbf{k} \sigma}^{t^{\prime} t}=\frac{1}{\Omega} \sqrt{2} \sum_{S \nu T q}(-1)^{\frac{1}{2}-\sigma^{\prime}}\left(\frac{1}{2} \sigma^{\prime} \frac{1}{2}-\sigma \mid S-\nu\right)(-1)^{q} \mathcal{T}_{T-q}^{t^{\prime} t} U_{S \nu}^{T q}(\mathbf{q}),
$$

where the abbreviation $\mathcal{T}_{T q}^{t^{\prime} t}=\left\langle\frac{1}{2} t^{\prime}\left|\mathcal{T}_{T q}\right| \frac{1}{2} t\right\rangle$ has been used for the isospin matrix element. We now insert Eq. 4.14 for $U_{S \nu}^{T q}(\mathbf{q})$, and we change the coupling order in the Clebsch-Gordan coefficient involving the target spin using

$$
\left(I_{i} M_{i} J M \mid I_{f} M_{f}\right)=(-1)^{I_{i}-M_{i}} \hat{J}^{-1} \hat{I}_{f}\left(I_{f} M_{f} I_{i}-M_{i} \mid J M\right) .
$$


The result is

$$
\begin{aligned}
T_{\mathbf{k}^{\prime} \sigma^{\prime}, \mathbf{k} \sigma}^{t^{\prime}}=\frac{1}{\Omega} & \sqrt{2} \sum_{\substack{L S J T \\
\mu \nu M q}}( \pm i)^{L}(-1)^{\frac{1}{2}-\sigma^{\prime}+I_{i}-M_{i}} \hat{J}^{-1} \hat{I}_{f} \\
& \times\left(I_{f} M_{f} I_{i}-M_{i} \mid J M\right)\left(\frac{1}{2} \sigma^{\prime} \frac{1}{2}-\sigma \mid S-\nu\right)(L \mu S \nu \mid J M) \\
& \times(-1)^{q} \mathcal{T}_{T-q}^{t^{\prime} t} U_{L S J}^{T q}(q) Y_{L \mu}^{*}(\hat{\mathbf{q}})
\end{aligned}
$$

We can make the notation a bit more compact by defining a radial transition potential between the projectile states with initial isospin projection $t$ and final projection $t^{\prime}$,

$$
U_{L S J}^{t^{\prime} t}=\sum_{T q}(-1)^{q} \mathcal{T}_{T-q}^{t^{\prime} t} U_{L S J}^{T q}
$$

so that we now have

$$
\begin{aligned}
T_{\mathbf{k}^{\prime} \sigma^{\prime}, \mathbf{k} \sigma}^{t^{\prime} t}=\frac{1}{\Omega} & \sqrt{2} \sum_{\substack{L S J \\
\mu \nu M}}( \pm i)^{L}(-1)^{\frac{1}{2}-\sigma^{\prime}+I_{i}-M_{i}} \hat{J}^{-1} \hat{I}_{f} \\
& \times\left(I_{f} M_{f} I_{i}-M_{i} \mid J M\right)\left(\frac{1}{2} \sigma^{\prime} \frac{1}{2}-\sigma \mid S-\nu\right)(L \mu S \nu \mid J M) \\
& \times U_{L S J}^{t^{\prime} t}(q) Y_{L \mu}^{*}(\hat{\mathbf{q}})
\end{aligned}
$$

We are free to choose the quantization axis for angular momentum along the momentumtransfer axis $\hat{\mathbf{q}}$, so that $Y_{L \mu}^{*}(\hat{\mathbf{q}}) \rightarrow \sqrt{(2 L+1) /(4 \pi)} \delta_{\mu 0}$. This eliminates the sum on $\mu$ and also on $M$, since $M=\nu$. Then the expression for the $T$-matrix becomes

$$
\begin{aligned}
T_{\mathbf{k}^{\prime} \sigma^{\prime}, \mathbf{k} \sigma}^{t^{\prime} t}=\frac{1}{\Omega} & \sqrt{\frac{2}{4 \pi}} \sum_{L S J \nu}( \pm i)^{L}(-1)^{\frac{1}{2}-\sigma^{\prime}+I_{i}-M_{i}} \hat{L} \hat{J}^{-1} \hat{I}_{f} \\
& \times\left(I_{f} M_{f} I_{i}-M_{i} \mid J \nu\right)\left(\frac{1}{2} \sigma^{\prime} \frac{1}{2}-\sigma \mid S-\nu\right)(L 0 S \nu \mid J \nu) U_{L S J}^{t^{\prime} t}(q)
\end{aligned}
$$

To determine scattering cross sections, the first step is to take the absolute square of the $T$-matrix element, then sum over final target substates and average over initial target substates. The factor $(-1)^{\frac{1}{2}-\sigma^{\prime}+I_{i}-M_{i}}$ does not depend on any of the summed quantum numbers $L S J \nu$, and therefore disappears when squared. When the sum is performed over $M_{i}$ and $M_{f}$ and the orthogonality relation for the Clebsch-Gordan coefficients containing these is invoked, we see that there is no interference between terms with different values of $J$ or $\nu$. Thus

$$
\begin{aligned}
& \frac{1}{2 I_{i}+1} \sum_{M_{i} M_{f}}\left|T_{\mathbf{k}^{\prime} \sigma^{\prime}, \mathbf{k} \sigma}^{t^{\prime} t}\right|^{2}=\frac{1}{\Omega^{2}} \frac{2}{4 \pi} \frac{2 I_{f}+1}{2 I_{i}+1} \\
& \quad \times \sum_{J \nu}\left|\sum_{L S}( \pm i)^{L} \hat{L} \hat{J}^{-1}\left(\frac{1}{2} \sigma^{\prime} \frac{1}{2}-\sigma \mid S-\nu\right)(L 0 S \nu \mid J \nu) U_{L S J}^{t^{\prime} t}(q)\right|^{2} .
\end{aligned}
$$

Similarly, we sum over final projectile spin substates $\sigma^{\prime}$ and average over initial substates $\sigma$. This procedure removes the interference between different values of $S$, yielding

$$
\mathcal{M}^{2} \equiv \frac{1}{2\left(2 I_{i}+1\right)} \sum_{M_{i} M_{f} \sigma \sigma^{\prime}}\left|T_{\mathbf{k}^{\prime} \sigma^{\prime}, \mathbf{k} \sigma}^{t^{\prime}}\right|^{2}=\frac{1}{\Omega^{2}} \frac{1}{4 \pi} \frac{2 I_{f}+1}{2 I_{i}+1}
$$




$$
\times \sum_{S J \nu}\left|\sum_{L}( \pm i)^{L} \hat{L} \hat{J}^{-1}(L 0 S \nu \mid J \nu) U_{L S J}^{t^{\prime} t}(q)\right|^{2}
$$

where we have defined $\mathcal{M}^{2}$ to describe the quantity we have calculated. The differential cross section is determined from $\mathcal{M}^{2}$ by

$$
\frac{d \sigma}{d \Omega}=\left(\frac{\mu}{2 \pi \hbar^{2}}\right)^{2} \frac{k^{\prime}}{k} \Omega^{2} \mathcal{M}^{2}
$$

where $\mu$ is the reduced mass of projectile and target. Upon inserting $\mathcal{M}^{2}$ from the plane-wave approximation, we find the result

$$
\frac{d \sigma}{d \Omega}=\frac{1}{4 \pi}\left(\frac{\mu}{2 \pi \hbar^{2}}\right)^{2} \frac{k^{\prime}}{k} \frac{2 I_{f}+1}{2 I_{i}+1} \sum_{S J \nu}\left|\sum_{L}( \pm i)^{L} \hat{L} \hat{J}^{-1}(L 0 S \nu \mid J \nu) U_{L S J}^{t^{\prime} t}(q)\right|^{2} .
$$

We make two observations that will further simplify the cross section formulae:

- Values of $L$ are limited to $J=0, \pm 1$. For a given $J$, if the parity change between the initial and final target states is $(-1)^{J}$, only $L=J$ contributes; this is called a natural parity transition. If the parity change is $(-1)^{J+1}$, then $L$ is restricted to the two values $L=J \pm 1$; these are the unnatural parity transitions. The amplitudes for these two classes cannot interfere.

- The spin-transfer projection $\nu$ appears only in the coefficient $(L 0 S \nu \mid J \nu)$. We can eliminate the terms with $\nu=-1$ by using the symmetry relation for inverting the m-values in the Clebsch-Gordan coefficient. A little thought shows that we can drop these terms if we multiply the cross section terms in Eq. 4.33 by $1+\delta_{\nu 1}$ or, equivalently, the amplitudes by the square root of this factor.

Based on the above, we define a factor

$$
F_{L S J \nu}^{\xi}=( \pm i)^{L-J+\xi} \sqrt{1+\delta_{\nu 1}} \hat{L} \hat{J}^{-1}(L 0 S \nu \mid J \nu)
$$

where $\xi=0$ for natural parity transitions, and $\xi=1$ for unnatural. All of the required values of $F_{L S J \nu}^{\xi}$ are given in Table 4.1, together with their component factors.

Using the above values for $F_{L S J \nu}^{\xi}$, the cross section can be expressed as

$$
\frac{d \sigma}{d \Omega}=\frac{1}{4 \pi}\left(\frac{\mu}{2 \pi \hbar^{2}}\right)^{2} \frac{k^{\prime}}{k} \frac{2 I_{f}+1}{2 I_{i}+1} \sum_{\substack{S J \\ \nu=0,1}}\left|\sum_{L} F_{L S J \nu}^{\xi} U_{L S J}^{t^{\prime} t}(q)\right|^{2}
$$

which can be separated into natural and unnatural parity terms as

$$
\begin{aligned}
& \frac{d \sigma}{d \Omega}=\frac{1}{4 \pi}\left(\frac{\mu}{2 \pi \hbar^{2}}\right)^{2} \frac{k^{\prime}}{k} \frac{2 I_{f}+1}{2 I_{i}+1} \\
& \quad \times \sum_{\substack{S J \\
\nu=0,1}}\left\{\left|F_{J S J \nu}^{\xi=0} U_{J S J}^{t^{\prime} t}(q)\right|^{2}+\left|F_{J-1 S J \nu}^{\xi=1} U_{J-1 S J}^{t^{\prime} t}(q)+F_{J+1 S J \nu}^{\xi=1} U_{J+1 S J}^{t^{\prime} t}(q)\right|^{2}\right\} .
\end{aligned}
$$




\begin{tabular}{|c|c|c|c|c|c|}
\hline \hline \multicolumn{7}{|c|}{ Natural parity $(\xi=0)$} \\
\hline$L S J \nu$ & $( \pm i)^{L-J+\xi}$ & $\sqrt{1+\delta_{\nu 0}}$ & $\hat{L} \hat{J}^{-1}$ & $(L 0 S \nu \mid J \nu)$ & $F_{L S J \nu}^{\xi}$ \\
\hline \hline$J 0 J 0$ & 1 & 1 & 1 & 1 & 1 \\
$J 1 J 1$ & 1 & $\sqrt{2}$ & 1 & $-1 / \sqrt{2}$ & -1 \\
\hline \hline \multicolumn{7}{|c|}{ Unnatural parity $(\xi=1)$} \\
\hline$L S J \nu$ & $( \pm i)^{L-J+\xi}$ & $\sqrt{1+\delta_{\nu 0}}$ & $\hat{L} \hat{J}^{-1}$ & $(L 0 S \nu \mid J \nu)$ & $F_{L S J \nu}^{\xi}$ \\
\hline \hline$(J-1) 1 J 0$ & 1 & 1 & $\sqrt{\frac{2 J-1}{2 J+1}}$ & $\sqrt{\frac{J}{2 J-1}}$ & $\sqrt{\frac{J}{2 J+1}}$ \\
$(J+1) 1 J 0$ & -1 & 1 & $\sqrt{\frac{2 J+3}{2 J+1}}$ & $-\sqrt{\frac{J+1}{2 J+3}}$ & $\sqrt{\frac{J+1}{2 J+1}}$ \\
$(J-1) 1 J 1$ & 1 & $\sqrt{2}$ & $\sqrt{\frac{2 J-1}{2 J+1}}$ & $\sqrt{\frac{J+1}{2(2 J-1)}}$ & $\sqrt{\frac{J+1}{2 J+1}}$ \\
$(J+1) 1 J 1$ & -1 & $\sqrt{2}$ & $\sqrt{\frac{2 J+3}{2 J+1}}$ & $\sqrt{\frac{J}{2(2 J+3)}}$ & $-\sqrt{\frac{J}{2 J+1}}$ \\
\hline
\end{tabular}

Table 4.1: Values and components of the amplitude factor $F_{L S J \nu}^{\xi}$ defined in Eq. 4.34.

To get to this equation from Eq. 4.33 using the above definition of $F_{L S J \nu}^{\xi}$, we have arbitrarily multiplied the amplitudes by a factor $( \pm i)^{-J+\xi}$. This is permissible since this factor yields 1 when its absolute square is taken.

For each value of $J$, we now explicitly carry out the sum over $S \nu$, for which the permitted values are $(00),(10),(11)$, using values of $F_{L S J \nu}^{\xi}$ from Table 4.1. In carrying out the sum, we note that $F_{J 1 J 0}^{\xi=0}=0$ because the Clebsch-Gordan coefficient $(J 010 \mid J 0)$ vanishes. For the unnatural-parity terms, we also define longitudinal $(\nu=0)$ and transverse $(\nu=1)$ components $U_{\| 1 J}^{t^{\prime} t}$ and $U_{\perp 1 J}^{t^{\prime} t}$, respectively, which are linear combinations of the $L=J-1$ and $L=J+1$ terms:

$$
\begin{aligned}
U_{\| 1 J}^{t^{\prime} t}(q) & =\sqrt{\frac{J}{2 J+1}} U_{J-11 J}^{t^{\prime} t}(q)+\sqrt{\frac{J+1}{2 J+1}} U_{J+11 J}^{t^{\prime} t}(q) \\
U_{\perp 1 J}^{t^{\prime} t}(q) & =\sqrt{\frac{J+1}{2 J+1}} U_{J-11 J}^{t^{\prime} t}(q)-\sqrt{\frac{J}{2 J+1}} U_{J+11 J}^{t^{\prime} t}(q),
\end{aligned}
$$

where we note that for $J=0, U_{\| 10}^{t^{\prime} t}=U_{110}^{t^{\prime} t}$ and $U_{\perp 10}^{t^{\prime} t}=0$.

Thus the cross section simplifies to

$$
\begin{aligned}
\frac{d \sigma}{d \Omega} & =\frac{1}{4 \pi}\left(\frac{\mu}{2 \pi \hbar^{2}}\right)^{2} \frac{k^{\prime}}{k} \frac{2 I_{f}+1}{2 I_{i}+1} \\
& \times \sum_{J=\left|I_{i}-I_{f}\right|}^{I_{i}+I_{f}}\left\{\left|U_{J 0 J}^{t^{\prime} t}(q)\right|^{2}+\left|U_{J 1 J}^{t^{\prime} t}(q)\right|^{2}+\left|U_{\| 1 J}^{t^{\prime} t}(q)\right|^{2}+\left|U_{\perp 1 J}^{t^{\prime} t}(q)\right|^{2}\right\} .
\end{aligned}
$$

The first two terms are natural parity, and the second two unnatural; for a given $J$ either one set or the other is present but not both. The second and fourth terms are forbidden for $J=0$. This cross section formula is consistent with the results of Eqs. 10,54,55 of Ref. [1], except that we have an extra factor of $k^{\prime} / k$ because we have not neglected energy loss in the collision, as was done in [1]. 
Finally, we return to the T-matrix expression of Eq. 4.29 and write out all of its terms for each $J$, which involves the same four $U$ quantities as in the cross section. This can be used as the starting point for calculating spin variables, although we will not pursue this. The result is

$$
\begin{aligned}
& T_{\mathbf{k}^{\prime} \sigma^{\prime}, \mathbf{k} \sigma}^{t^{\prime} t}=\frac{1}{\Omega} \sqrt{\frac{1}{4 \pi}}(-1)^{\frac{1}{2}-\sigma^{\prime}+I_{i}-M_{i}} \sqrt{2 I_{f}+1} \sum_{J=\left|J_{i}-J_{f}\right|}^{J_{i}+J_{f}} \\
& \sqrt{2}( \pm i)^{J} \quad\left(I_{f} M_{f} I_{i} M_{i} \mid J 0\right) \quad\left(\frac{1}{2} \sigma^{\prime} \frac{1}{2}-\sigma \mid 00\right) \quad U_{J 0 J}^{t^{\prime} t}(q)
\end{aligned}
$$

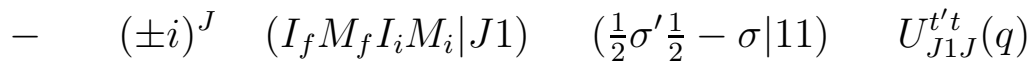

$$
\begin{aligned}
& +\quad( \pm i)^{J} \quad\left(I_{f} M_{f} I_{i} M_{i} \mid J-1\right)\left(\frac{1}{2} \sigma^{\prime} \frac{1}{2}-\sigma \mid 1-1\right) U_{J 1 J}^{t^{\prime} t}(q) \\
& +\quad( \pm i)^{J-1}\left(I_{f} M_{f} I_{i} M_{i} \mid J 1\right) \quad\left(\frac{1}{2} \sigma^{\prime} \frac{1}{2}-\sigma \mid 11\right) \quad U_{\perp 1 J}^{t^{\prime} t}(q) \\
& +\sqrt{2}( \pm i)^{J-1}\left(I_{f} M_{f} I_{i} M_{i} \mid J 0\right) \quad\left(\frac{1}{2} \sigma^{\prime} \frac{1}{2}-\sigma \mid 10\right) \quad U_{\| 1 J}^{t^{\prime} t}(q) \\
& +\quad( \pm i)^{J-1}\left(I_{f} M_{f} I_{i} M_{i} \mid J-1\right)\left(\frac{1}{2} \sigma^{\prime} \frac{1}{2}-\sigma \mid 1-1\right) U_{\perp 1 J}^{t^{\prime} t}(q),
\end{aligned}
$$

where the first three terms are natural parity and the last three unnatural parity. As in the cross section expression, terms containing $U_{J 1 J}^{t^{\prime} t}$ and $U_{\perp 1 J}^{t^{\prime} t}$ are forbidden for $J=0$.

By using the defining relation Eq. 4.27, we can express the transition-potential functions $U^{t^{\prime} t}$ in terms of their isospin representation $U^{T q}$ by

$$
U^{t^{\prime} t}=\mathcal{T}_{00}^{t^{\prime} t} U^{00}-\mathcal{T}_{1-1}^{t^{\prime} t} U^{11}+\mathcal{T}_{10}^{t^{\prime} t} U^{10}-\mathcal{T}_{11}^{t^{\prime} t} U^{1-1} .
$$

This relation is valid for all of the possible subscripts that may appear on the quantities $U$. The expression is evaluated for all combinations of $t^{\prime}, t$ in the fourth column of Table 4.2, with an indication of a possible reaction for each combination in the third column. Values of the $\mathcal{T}_{T q}^{t^{\prime} t}$ coefficients were taken from Table B.1. In the last column of Table 4.2 the radial momentum-space potentials are expressed using products of effective interaction and densities, according to the definition of Eq. 4.15 and the neutron, proton, and charge-exchange densities of Eqs. 3.18-3.21. We have shown only the isospin index $T$ on the interaction; the spin index $S$ is suppressed.

\begin{tabular}{|rrccr|}
\hline$t^{\prime}$ & $t$ & Reaction & $U^{t^{\prime} t}$ & $U^{t^{\prime} t}(q) /(4 \pi)$ \\
\hline$\frac{1}{2}$ & $\frac{1}{2}$ & $(\mathrm{n}, \mathrm{n})$ & $U^{00}+U^{10}$ & {$\left[v^{0}(q)+v^{1}(q)\right] \rho^{n}(q)+\left[v^{0}(q)-v^{1}(q)\right] \rho^{p}(q)$} \\
$\frac{1}{2}$ & $-\frac{1}{2}$ & $(\mathrm{p}, \mathrm{n})$ & $\sqrt{2} U^{1-1}$ & $2 v^{1}(q) \rho^{p-n}(q)$ \\
$-\frac{1}{2}$ & $\frac{1}{2}$ & $(\mathrm{n}, \mathrm{p})$ & $-\sqrt{2} U^{11}$ & $2 v^{1}(q) \rho^{n \leftarrow p}(q)$ \\
$-\frac{1}{2}$ & $-\frac{1}{2}$ & $(\mathrm{p}, \mathrm{p})$ & $U^{00}-U^{10}$ & {$\left[v^{0}(q)-v^{1}(q)\right] \rho^{n}(q)+\left[v^{0}(q)+v^{1}(q)\right] \rho^{p}(q)$} \\
\hline
\end{tabular}

Table 4.2: The potentials $U^{t^{\prime} t}$ for a reaction with a projectile having initial isospin projection $t$ and final projection $t^{\prime}$, expressed in terms of the isospin potentials $U^{T q}$ and also the underlying effective interactions and densities. See text for details. Typical reactions are indicated in the third column. 


\section{Chapter 5}

\section{Coupled Channels Method}

We restrict our attention to inelastic scattering in which the composition and internal structure of the projectile are unchanged during the scattering. This avoids complications involving the orthogonality of the coupled channels. The following development also applies to charge-exchange reactions with minor modifications.

\subsection{Derivation of Radial Coupled Equations}

We need to find solutions to the Schrödinger equation

$$
(E-H)|\Psi\rangle=0,
$$

subject to appropriate boundary conditions. We expand the wave function in basis states that are eigenfunctions of the angular momentum of the entire projectile-target system. To do this, we define the part of the wave function in the channel $c$ as

$$
\frac{u_{c}(r)}{r}\left|\gamma_{c}\left\{\left[\left(l_{c} s_{c}\right) j_{c}\right] I_{c}\right\} \mathcal{J \mathcal { M }} ; t_{c}\right\rangle \equiv \frac{u_{c}(r)}{r}|c \mathcal{J M}\rangle,
$$

where we explicitly separate out the radial wave function $u_{c}$ in the coordinate between the center of masses of the target and projectile, and the ket represents all other variables. The channel index $c$ on the right represents all quantum numbers characterizing the system except $\mathcal{J}$ and $\mathcal{M}$. $l_{c}$ is the relative orbital angular momentum of target and projectile; $s_{c}$ is the projectile spin; $j_{c}$ is the vector coupling of $l_{c}$ and $s_{c} ; I_{c}$ is the spin of the target state, and $j_{c}$ and $I_{c}$ are coupled to total angular momentum $\mathcal{J}$. $t_{c}$ is the isospin projection $\left( \pm \frac{1}{2}\right)$ for the projectile; we consider only isospin $\frac{1}{2}$ particles. For projectiles of other isospin this quantum number should be left out or the treatment appropriately generalized. $\gamma_{c}$ represents all remaining quantum numbers necessary to completely specify the system, and may be viewed as an abbreviation for the product $\gamma_{c}=\alpha_{c} \beta_{c}$, where $\alpha_{c}$ and $\beta_{c}$ refer to target and projectile quantum numbers, respectively. The coupling order for the angular momenta as described in Eq. 5.2 is illustrated in Fig. 5.1.

The above description of the wave function in channel $c$ implicitly includes a factor $i^{l_{c}}$; that is, the orbital part of the wave function indicated by $l_{c}$ has the coordinate-space representation

$$
\left\langle\hat{\mathbf{r}} \mid l_{c} m_{c}\right\rangle=i^{l_{c}} Y_{l_{c} m_{c}}(\hat{\mathbf{r}}) .
$$




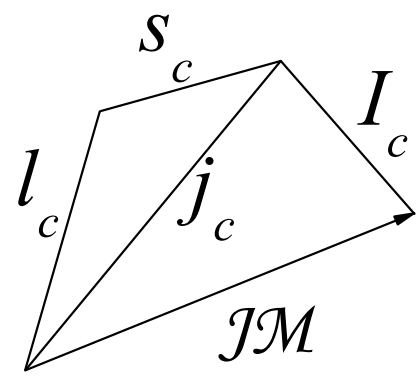

Figure 5.1: Coupling order for angular momenta in coupled-channels equations.

The $i^{l_{c}}$ factor is essential to the description of a scattering wave function, and must therefore be included either implicitly as in the present treatment or shown explicitly.

The internal state vectors $\left|s_{c} \sigma_{c}\right\rangle$ and $\left|I_{c} M_{c}\right\rangle$ of the projectile and target may optionally be defined with phases so that they have the simple time reversal properties

$$
\mathcal{T}\left|s_{c} \sigma_{c}\right\rangle=(-1)^{s_{c}+\sigma_{c}}\left|s_{c}-\sigma_{c}\right\rangle \quad \text { and } \quad \mathcal{T}\left|I_{c} M_{c}\right\rangle=(-1)^{I_{c}+M_{c}}\left|I_{c}-M_{c}\right\rangle,
$$

where $\mathcal{T}$ is the time reversal operator. If these phases are chosen and the $i^{l_{c}}$ factor is included with $Y_{l_{c} m_{c}}$, the complete states have the same time reversal properties:

$$
\mathcal{T}|c \mathcal{J \mathcal { M }}\rangle=(-1)^{\mathcal{J}+\mathcal{M}}|c \mathcal{J}-\mathcal{M}\rangle \text {. }
$$

In Eqs. 5.4 and 5.5 we have used the convention that a plus sign is used in the phase factors; others use a minus sign.

For a solution of Eq. 5.1 that is an eigenfunction of $\mathcal{J}$ and $\mathcal{M}$, we expand

$$
|\Psi\rangle=\sum_{c^{\prime}} \frac{u_{c^{\prime}}(r)}{r}\left|c^{\prime} \mathrm{J \mathcal {M }}\right\rangle .
$$

We insert this in Eq. 5.1 and operate on the left by $\langle c \mathcal{J \mathcal { M }}|$. This yields

$$
\left(E-H_{c c}^{\mathfrak{J}}\right) \frac{u_{c}(r)}{r}=\sum_{c^{\prime} \neq c} H_{c c^{\prime}}^{\mathfrak{J}} \frac{u_{c^{\prime}}(r)}{r},
$$

where

$$
H_{c c^{\prime}}^{\mathcal{J}}=\left\langle c \mathcal{J} \mathcal{M}|H| c^{\prime} \mathcal{J} \mathcal{M}\right\rangle
$$

which is independent of $\mathcal{M}$ because $H$ is rotationally invariant. Eq. 5.7 represents a set of coupled channels, which we now put in a more familiar form.

We write $H_{c c^{\prime}}^{\mathcal{J}}$ as

$$
\begin{aligned}
H_{c c^{\prime}}^{\mathfrak{J}} & =\left\langle c \mathcal{J \mathcal { M }}\left|H^{i n t}+T+V\right| c^{\prime} \mathcal{J \mathcal { M }}\right\rangle \\
& =\left\{E_{c}^{e x}-\frac{\hbar^{2}}{2 \mu} \frac{1}{r^{2}}\left(\frac{d}{d r} r^{2} \frac{d}{d r}\right)+\frac{l_{c}\left(l_{c}+1\right) \hbar^{2}}{2 \mu r^{2}}\right\} \delta_{c c^{\prime}}+V_{c c^{\prime}}^{\mathfrak{J}},
\end{aligned}
$$


where $H^{i n t}$ is the Hamiltonian for the internal energy of the projectile and target, $E_{c}^{e x}$ is the combined excitation energy of the projectile and target in channel $c$, and $\mu_{c}$ is the reduced mass of projectile and target. We insert this expression in Eq. 5.7 and multiply by $r$ from the left. If the potential term $V_{c c^{\prime}}^{\mathfrak{d}}$ is local and not explicitly momentum dependent, this yields

$$
\left\{\varepsilon_{c}+\frac{\hbar^{2}}{2 \mu_{c}} \frac{d^{2}}{d r^{2}}-\frac{l_{c}\left(l_{c}+1\right) \hbar^{2}}{2 \mu_{c} r^{2}}-V_{c c}^{\mathcal{J}}(r)\right\} u_{c}(r)=\sum_{c^{\prime} \neq c} V_{c c^{\prime}}^{\mathfrak{J}}(r) u_{c^{\prime}}(r),
$$

where $\varepsilon_{c}=E-E_{c}^{e x}$. In the usual case of a projectile incident on a specific excited state of the target (which is not necessarily the ground state), we shift the overall energy scale so that $\varepsilon_{c}=0$ for those channels $c$ in which the target is in this state. An alternative form for the coupled channel equations is

$$
\left\{\frac{d^{2}}{d r^{2}}-\frac{l_{c}\left(l_{c}+1\right)}{r^{2}}-\frac{2 \mu_{c}}{\hbar^{2}} V_{c c}^{\mathcal{J}}(r)+k_{c}^{2}\right\} u_{c}(r)=\sum_{c^{\prime} \neq c} \frac{2 \mu_{c}}{\hbar^{2}} V_{c c^{\prime}}^{\mathcal{J}}(r) u_{c^{\prime}}(r),
$$

where $k_{c}^{2}=2 \mu_{c} \varepsilon_{c} / \hbar^{2}$. If $V_{c c^{\prime}}^{\mathcal{J}}$ is nonlocal or momentum dependent (which may happen for either the diagonal or off-diagonal terms), we make the replacement

$$
V_{c c^{\prime}}^{\mathcal{J}} u_{c^{\prime}} \rightarrow r V_{c c^{\prime}}^{\mathcal{J}} \frac{u_{c^{\prime}}}{r}
$$

\subsection{Evaluation of Coupling Potentials}

We need to evaluate

$$
V_{c c^{\prime}}^{\mathcal{J}}(r)=\left\langle c \mathcal{J \mathcal { M }}|V| c^{\prime} \mathcal{J \mathcal { M }}\right\rangle,
$$

where all coordinates are integrated (or summed) over except the radial target-projectile distance $r$. We assume that $V_{c c^{\prime}}^{\mathcal{J}}$ as well as the two-body interaction between the target nucleons and the projectile are local; this is not a serious restriction in the following development. The potential $V_{c c^{\prime}}^{\mathcal{J}}$ is clearly independent of $\mathcal{M}$. If the states and the interaction have the behavior under time reversal (see Eq. 5.5) that

$$
\mathcal{T}|c \mathcal{J \mathcal { M }}\rangle=(-1)^{\mathcal{J} \pm \mathcal{M}}|c \mathcal{J}-\mathcal{M}\rangle \quad \text { and } \quad \mathcal{T} V \mathcal{T}^{-1}=V^{\dagger},
$$

then it is easily shown that the coupling potential is symmetric: $V_{c^{\prime} c}^{\mathfrak{d}}=V_{c c^{\prime}}^{\mathfrak{d}}$.

We treat the central spin- and isospin-dependent two-body interaction of Eq. 2.10, which after summing over target nucleons is

$$
V=\sum_{L S J T} \sum_{n} v_{L}^{S T}\left(r, r_{n}\right)(-1)^{L+S-J}\left[Y_{L}(\hat{\mathbf{r}}) \times \mathcal{S}_{S}\right]_{J} \cdot\left[Y_{L}\left(\hat{\mathbf{r}}_{n}\right) \times \mathcal{S}_{S}^{n}\right]_{J} \quad \mathcal{T}_{T} \cdot \mathcal{T}_{T}^{n}
$$

We put this expression in Eq. 5.14 and expand the description of the states to get

$$
\begin{aligned}
& V_{c c^{\prime}}^{\mathcal{J}}(r)=\left\langle\gamma_{c}\left\{\left[\left(l_{c} s_{c}\right) j_{c}\right] I_{c}\right\} \mathcal{J \mathcal { M }} ; t_{c}\right| \sum_{L S J T} \sum_{n} v_{L}^{S T}\left(r, r_{n}\right)(-1)^{L+S-J}
\end{aligned}
$$

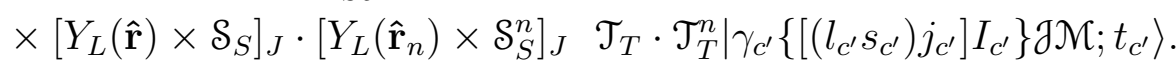


We can use the expression for matrix elements of a scalar product of tensor operators (e.g. Edmonds [2], p. 111 Eq. 7.1.6) to put this in the form of a sum of products of matrix elements in the projectile and target spaces,

$$
\begin{aligned}
& V_{c c^{\prime}}^{\mathcal{J}}(r)=\sum_{L S J T q}(-1)^{j_{c^{\prime}}+I_{c}+\mathcal{J}}\left\{\begin{array}{lll}
\mathcal{J} & I_{c} & j_{c} \\
J & j_{c^{\prime}} & I_{c^{\prime}}
\end{array}\right\}(-1)^{L+S-J} \\
& \times\left(\beta_{c}\left(l_{c} s_{c}\right) j_{c} ; t_{c}\left\|\left[Y_{L}(\hat{\mathbf{r}}) \times \mathcal{S}_{S}\right]_{J} \mathcal{T}_{T q}^{\dagger}\right\| \beta_{c^{\prime}}\left(l_{c^{\prime}} s_{c^{\prime}}\right) j_{c^{\prime}} ; t_{c^{\prime}}\right) \\
& \times\left(\alpha_{c} I_{c}\left\|\sum_{n} v_{L}^{S T}\left(r, r_{n}\right)\left[Y_{L}\left(\hat{\mathbf{r}}_{n}\right) \times \mathcal{S}_{S}^{n}\right]_{J} \mathcal{T}_{T q}^{n}\right\| \alpha_{c^{\prime}} I_{c^{\prime}}\right),
\end{aligned}
$$

where the quantity $\mathcal{T}_{T q}^{\dagger}$ appearing in the first (projectile) matrix element is $(-1)^{q} \mathcal{T}_{T-q}$.

We examine the second reduced matrix element, which is the one in the target space. We find

$$
\begin{aligned}
& \left(\alpha_{c} I_{c}\left\|\sum_{n} v_{L}^{S T}\left(r, r_{n}\right)\left[Y_{L}\left(\hat{\mathbf{r}}_{n}\right) \times \mathcal{S}_{S}^{n}\right]_{J} \mathcal{T}_{T q}^{n}\right\| \alpha_{c^{\prime}} I_{c^{\prime}}\right) \\
& \quad=\int_{0}^{\infty} d r_{t} r_{t}^{2} v_{L}^{S T}\left(r, r_{t}\right)\left(\alpha_{c} I_{c}\left\|\sum_{n} \frac{\delta\left(r_{t}-r_{n}\right)}{r_{t} r_{n}}\left[Y_{L}\left(\hat{\mathbf{r}}_{n}\right) \times \mathcal{S}_{S}^{n}\right]_{J} \mathcal{T}_{T q}^{n}\right\| \alpha_{c^{\prime}} I_{c^{\prime}}\right) \\
& \quad \equiv 4 \pi \sqrt{2 I_{c}+1} \int_{0}^{\infty} d r_{t} r_{t}^{2} v_{L}^{S T}\left(r, r_{t}\right) \rho_{L S J}^{T q, c c^{\prime}}\left(r_{t}\right)
\end{aligned}
$$

where in the last line we have defined the coordinate-space radial transition density,

$$
\rho_{L S J}^{T q, c c^{\prime}}\left(r_{t}\right)=\frac{1}{\sqrt{2 I_{c}+1}}\left(\alpha_{c} I_{c}\left\|\sum_{n} \frac{1}{4 \pi} \frac{\delta\left(r_{t}-r_{n}\right)}{r_{t} r_{n}}\left[Y_{L}\left(\hat{\mathbf{r}}_{n}\right) \times \mathcal{S}_{S}^{n}\right]_{J} \mathcal{T}_{T q}^{n}\right\| \alpha_{c^{\prime}} I_{c^{\prime}}\right)
$$

which is the same definition as in Eq. 3.11. Alternatively, we can insert the expression for $v_{L}^{S T}\left(r, r_{n}\right)$ from Eq. 2.17 directly into the reduced matrix element, and find the following expression involving momentum-space quantities:

$$
\begin{aligned}
& \left(\alpha_{c} I_{c}\left\|\sum_{n} v_{L}^{S T}\left(r, r_{n}\right)\left[Y_{L}\left(\hat{\mathbf{r}}_{n}\right) \times \mathcal{S}_{S}^{n}\right]_{J} \mathcal{T}_{T q}^{n}\right\| \alpha_{c^{\prime}} I_{c^{\prime}}\right) \\
& \quad=\frac{2}{\pi} \int_{0}^{\infty} d q q^{2} j_{L}(q r) v^{S T}(q)\left(\alpha_{c} I_{c}\left\|\sum_{n} j_{L}\left(q r_{n}\right)\left[Y_{L}\left(\hat{\mathbf{r}}_{n}\right) \times \mathcal{S}_{S}^{n}\right]_{J} \mathcal{T}_{T q}^{n}\right\| \alpha_{c^{\prime}} I_{c^{\prime}}\right) \\
& \quad \equiv \sqrt{2 I_{c}+1} \frac{2}{\pi} \int_{0}^{\infty} d q q^{2} j_{L}(q r) v^{S T}(q) \rho_{L S J}^{T q, c c^{\prime}}(q)
\end{aligned}
$$

where, in agreement with Eq. 3.14, we have defined the momentum-space radial transition density in the last line,

$$
\rho_{L S J}^{T q, c c^{\prime}}(q)=\frac{1}{\sqrt{2 I_{c}+1}}\left(\alpha_{c} I_{c}\left\|\sum_{n} j_{L}\left(q r_{n}\right)\left[Y_{L}\left(\hat{\mathbf{r}}_{n}\right) \times \mathcal{S}_{S}^{n}\right]_{J} \mathcal{T}_{T q}^{n}\right\| \alpha_{c^{\prime}} I_{c^{\prime}}\right),
$$

We define the quantity $U_{L S J}^{T q, c c^{\prime}}$ by

$$
\left(\alpha_{c} I_{c}\left\|\sum_{n} v_{L}^{S T}\left(r, r_{n}\right)\left[Y_{L}\left(\hat{\mathbf{r}}_{n}\right) \times \mathcal{S}_{S}^{n}\right]_{J} \mathcal{T}_{T q}^{n}\right\| \alpha_{c^{\prime}} I_{c^{\prime}}\right)=\sqrt{2 I_{c}+1} U_{L S J}^{T q, c c^{\prime}}(r)
$$


and from Eqs. 5.20 and 5.23 we find

$$
\begin{aligned}
U_{L S J}^{T q, c c^{\prime}}(r) & =\frac{2}{\pi} \int_{0}^{\infty} d q q^{2} j_{L}(q r) v^{S T}(q) \rho_{L S J}^{T q, c c^{\prime}}(q) \\
& =4 \pi \int_{0}^{\infty} d r_{t} r_{t}^{2} v_{L}^{S T}\left(r, r_{t}\right) \rho_{L S J}^{T q, c c^{\prime}}\left(r_{t}\right)
\end{aligned}
$$

These expressions are exactly the same as those found in the discussion of the distorted-wave approximation, namely Eqs. 4.10 and 4.9. Although we will not need it for present purposes, it is instructive to take the Fourier transform of Eq. 5.26, using Eqs. C.7 and C.56. The result,

$$
U_{L S J}^{T q, c c^{\prime}}(q)=4 \pi v^{S T}(q) \rho_{L S J}^{T q, c c^{\prime}}(q),
$$

is in agreement with Eq. 4.15 .

Eqs. 5.25-5.27 completely specify the target-space reduced matrix element in terms of the effective interaction and transition density evaluated in either momentum or coordinate space.

We next evaluate the projectile-space matrix element in Eq. 5.18. The first step is to factor out the isospin matrix element, so that we have

$$
\begin{aligned}
& \left(\beta_{c}\left(l_{c} s_{c}\right) j_{c} ; t_{c}\left\|\left[Y_{L}(\hat{\mathbf{r}}) \times \mathcal{S}_{S}\right]_{J} \mathcal{T}_{T q}^{\dagger}\right\| \beta_{c^{\prime}}\left(l_{c^{\prime}} s_{c^{\prime}}\right) j_{c^{\prime}} ; t_{c^{\prime}}\right) \\
& =\left(\beta_{c}\left(l_{c} s_{c}\right) j_{c}\left\|\left[Y_{L}(\hat{\mathbf{r}}) \times \mathcal{S}_{S}\right]_{J}\right\| \beta_{c^{\prime}}\left(l_{c^{\prime}} s_{c^{\prime}}\right) j_{c^{\prime}}\right)(-1)^{q} \mathcal{T}_{T-q}^{t_{c} t_{c^{\prime}}},
\end{aligned}
$$

where as usual $\mathcal{T}_{T-q}^{t_{c} t_{c^{\prime}}}=\left\langle\frac{1}{2} t_{c}\left|\mathcal{T}_{T-q}\right| \frac{1}{2} t_{c^{\prime}}\right\rangle$. Using Edmonds, Eq. 7.1.5, p. 110, the matrix element on the right can be expressed as

$$
\begin{aligned}
\left(\beta_{c}\left(l_{c} s_{c}\right) j_{c} \|\left[Y_{L}\right.\right. & \left.\left.\times \mathcal{S}_{S}\right]_{J} \| \beta_{c^{\prime}}\left(l_{c^{\prime}} s_{c^{\prime}}\right) j_{c^{\prime}}\right) \\
& =\hat{j_{c}} \hat{j_{c^{\prime}}} \hat{J}\left\{\begin{array}{ccc}
l_{c} & l_{c^{\prime}} & L \\
s_{c} & s_{c^{\prime}} & S \\
j_{c} & j_{c^{\prime}} & J
\end{array}\right\}\left(l_{c}\left\|Y_{L}\right\| l_{c^{\prime}}\right)\left(\beta_{c} s_{c}\left\|\mathcal{S}_{S}\right\| \beta_{c^{\prime}} s_{c^{\prime}}\right),
\end{aligned}
$$

and the orbital matrix element is evaluated using Edmonds, Eqs. 5.4.4, p. 76 as

$$
\left(l_{c}|| Y_{L}|| l_{c^{\prime}}\right)=i^{l_{c^{\prime}}-l_{c}}(-1)^{l_{c}} \frac{1}{\sqrt{4 \pi}} \hat{l_{c}} \hat{l_{c^{\prime}}} \hat{L}\left(\begin{array}{ccc}
l_{c} & L & l_{c^{\prime}} \\
0 & 0 & 0
\end{array}\right),
$$

where we have now made the $i^{l}$ factors in each channel explicit (see Eq. 5.3). The result is

$$
\begin{aligned}
\left(\beta_{c}\left(l_{c} s_{c}\right) j_{c} \|\left[Y_{L}\right.\right. & \left.\left.\times \mathcal{S}_{S}\right]_{J} \| \beta_{c^{\prime}}\left(l_{c^{\prime}} s_{c^{\prime}}\right) j_{c^{\prime}}\right)=i^{l_{c^{\prime}}}-l_{c} \frac{1}{\sqrt{4 \pi}}(-1)^{l_{c}} \hat{l_{c}} \hat{l_{c^{\prime}}} \hat{j_{c}} \hat{j_{c^{\prime}}} \hat{L} \hat{J} \\
& \times\left(\begin{array}{ccc}
l_{c} & l_{c^{\prime}} & L \\
0 & 0 & 0
\end{array}\right)\left\{\begin{array}{ccc}
l_{c} & l_{c^{\prime}} & L \\
s_{c} & s_{c^{\prime}} & S \\
j_{c} & j_{c^{\prime}} & J
\end{array}\right\}\left(\beta_{c} s_{c}\left\|\mathcal{S}_{S}\right\| \beta_{c^{\prime}} s_{c^{\prime}}\right) .
\end{aligned}
$$

We are now in a position to assemble the complete expression for the coupling potential. As was done in Eq. 4.27 we define a radial transition potential that sums over isospin,

$$
U_{L S J}^{c c^{\prime}}=\sum_{T q}(-1)^{q} \mathcal{T}_{T-q}^{t_{c t} t_{c^{\prime}}} U_{L S J}^{T q, c c^{\prime}}
$$


and we insert quantities from this and Eqs. 5.25 and 5.32 into Eq. 5.18 to obtain

$$
\begin{aligned}
& V_{c c^{\prime}}^{\mathcal{J}}(r)=i^{l_{c^{\prime}}-l_{c}} \sum_{L S J} \\
& \quad \times \frac{1}{\sqrt{4 \pi}}(-1)^{j_{c^{\prime}}+I_{c}+\mathcal{J}+l_{c}} \hat{l_{c}} \hat{l_{c^{\prime}}} \hat{j_{c}} \hat{j_{c^{\prime}}} \hat{L} \hat{J}\left\{\begin{array}{ccc}
j_{c} & j_{c^{\prime}} & J \\
I_{c^{\prime}} & I_{c} & \mathcal{J}
\end{array}\right\}\left(\begin{array}{ccc}
l_{c} & l_{c^{\prime}} & L \\
0 & 0 & 0
\end{array}\right)\left\{\begin{array}{ccc}
l_{c} & l_{c^{\prime}} & L \\
s_{c} & s_{c^{\prime}} & S \\
j_{c} & j_{c^{\prime}} & J
\end{array}\right\} \\
& \times(-1)^{L+S-J}\left(\beta_{c} s_{c}\left\|\mathcal{S}_{S}\right\| \beta_{c^{\prime}} S_{c^{\prime}}\right) \sqrt{2 I_{c}+1} U_{L S J}^{c c^{\prime}}(r) .
\end{aligned}
$$

If we designate the second line by $F_{L S J}^{\mathcal{J}}\left(l_{c} s_{c} j_{c} I_{c} ; l_{c^{\prime}} s_{c^{\prime}} j_{c^{\prime}} I_{c^{\prime}}\right)$, the coupling potential can be written more compactly as

$$
\begin{aligned}
V_{c c^{\prime}}^{\mathcal{J}}(r)= & i^{l_{c^{\prime}}-l_{c}} \sum_{L S J} F_{L S J}^{\mathcal{J}}\left(l_{c} s_{c} j_{c} I_{c} ; l_{c^{\prime}} s_{c^{\prime}} j_{c^{\prime}} I_{c^{\prime}}\right) \\
& \times(-1)^{L+S-J}\left(\beta_{c} s_{c}\left\|\mathcal{S}_{S}\right\| \beta_{c^{\prime}} s_{c^{\prime}}\right) \sqrt{2 I_{c}+1} U_{L S J}^{c c^{\prime}}(r) .
\end{aligned}
$$

For the problem at hand the projectile spin and internal properties do not change because of the interaction, apart from (possibly) the isospin projection, which is included in the $U_{L S J}^{c c^{\prime}}$ factor. Thus $s_{c}=s_{c^{\prime}} \equiv s$ and we can drop the $\beta$ indices. For spin- $\frac{1}{2}$ particles we have

$$
\left(s=\frac{1}{2}\left\|\mathcal{S}_{S}\right\| s=\frac{1}{2}\right)=\sqrt{2(2 S+1)},
$$

and for spin-0 particles we have only $S=0$ and therefore $L=J$; we drop the spin- and isospin-dependent terms in the effective interaction and use

$$
\left(s=0\left\|\mathcal{S}_{0}\right\| s=0\right)=1
$$




\section{Chapter 6}

\section{Implementation and Computer Codes}

In this chapter we give general parameterizations for the coupling potentials used in distortedwave and coupled-channels calculations. Specific implementations are then described by how they relate to these general forms. We also describe the relation between the formulas for densities and interactions and their implementation in folding codes to yield plane-wave approximation cross sections and transition potentials.

\subsection{Coupled-Channels Method}

\subsubsection{General Forms for Coupling Potentials}

The coupling potentials used in coupled-channel calculations are the quantities $V_{c c^{\prime}}^{\mathcal{J}}(r)$ that appear on the right-hand side of the following system of coupled equations, which are described in Sec. 5.1:

$$
\left\{\frac{d^{2}}{d r^{2}}-\frac{l_{c}\left(l_{c}+1\right)}{r^{2}}-\frac{2 \mu_{c}}{\hbar^{2}} V_{c c}^{\mathfrak{J}}(r)+k_{c}^{2}\right\} u_{c}(r)=\sum_{c^{\prime} \neq c} \frac{2 \mu_{c}}{\hbar^{2}} V_{c c^{\prime}}^{\mathcal{J}}(r) u_{c^{\prime}}(r) .
$$

The notation is described in Sec. 5.1. We consider only local coupling potentials, but the generalization to nonlocal couplings is straightforward. The complete state vector in channel $c$ is

$$
\frac{u_{c}(r)}{r}\left|\gamma_{c}\left\{\left[\left(l_{c} s_{c}\right) j_{c}\right] I_{c}\right\} \mathcal{J \mathcal { M }}\right\rangle \equiv \frac{u_{c}(r)}{r}|c \mathcal{J} \mathcal{M}\rangle,
$$

where we do not show isospin quantum numbers explicitly. A representation of the channel-c state vector, except for its radial dependence, is

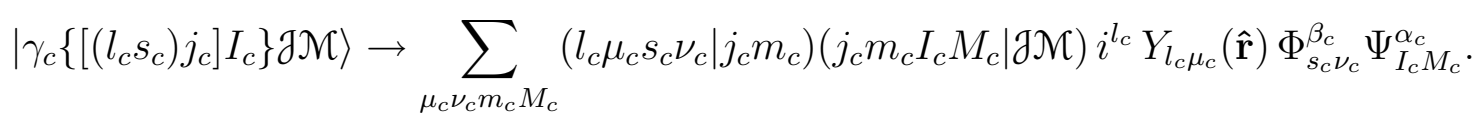

The quantities $\Phi_{s_{c} \nu_{c}}^{\beta_{c}}$ and $\Psi_{I_{c} M_{c}}^{\alpha_{c}}$ are the wave functions for the projectile and target, respectively; they are functions of the internal coordinates of these particles only. $\beta_{c}$ and $\alpha_{c}$ describe additional quantum numbers required to complete the description of the states of the projectile and target, and $\gamma_{c}$ is an abbreviation for the set $\beta_{c} \alpha_{c}$. Important properties of the description of the channel wave functions and the coupling potentials that need to be checked in intercomparisons between authors are 
- Inclusion of the phase factor $i^{l_{c}}$ along with $Y_{l_{c} \mu_{c}}$, inside the channel vector as is done here. This factor is present but inconspicuous when the channel vector is written as $|c \mathcal{J \mathcal { M }}\rangle$. In the potential, the corresponding phase factor $i^{l_{c^{\prime}}-l_{c}}$ is sometimes included in the potential (as here), but may be taken out of the potential and treated separately.

- Normalization of the angle factor, chosen here as $Y_{l_{c} \mu_{c}}(\hat{\mathbf{r}})$.

- Order of angular momentum coupling.

- The convention for defining the reduced matrix elements.

- Phases chosen for the angle factor and the target and projectile wave functions, which if appropriately chosen lead to simple time-reversal behavior for the entire state (see Eqs. 5.4,5.5).

- Direction of the vector between the target and the projectile. Unless otherwise stated, in this document the vector extends from the target to the projectile.

We consider a coupling of greater generality than in Sec. 5.2, which does not restrict the projectile spin and allows projectile excitation in principle. We do not include isospin operators explicitly, but the treatment of inelastic scattering and charge exchange in Sec. 5.2 and elsewhere in this report shows how this can be done. The operator, which is rotationally invariant, is

$$
\sum_{L S J}\left[Y_{L}(\hat{\mathbf{r}}) \times Q_{S}^{(p)}\right]_{J} \cdot Q_{J}^{(t)}
$$

where $Q_{S}^{(p)}$ and $Q_{J}^{(t)}$ are tensor operators of rank $S$ and $J$ in the projectile and target spaces, respectively. These tensor operators implicitly contain dependence on the radial coordinate $r$. We use the dot-product notation as shown above, but one occasionally encounters the vector coupling notation

$$
\sum_{L S J}\left[\left[Y_{L}(\hat{\mathbf{r}}) \times Q_{S}^{(p)}\right]_{J} \times Q_{J}^{(t)}\right]_{00}
$$

The connection between these is

$$
\left[Y_{L}(\hat{\mathbf{r}}) \times Q_{S}^{(p)}\right]_{J} \cdot Q_{J}^{(t)}=(-1)^{J} \sqrt{2 J+1}\left[\left[Y_{L}(\hat{\mathbf{r}}) \times Q_{S}^{(p)}\right]_{J} \times Q_{J}^{(t)}\right]_{00} .
$$

Thus the coupling potential is

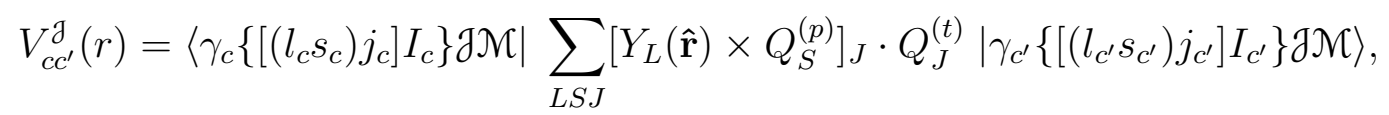

which can be written using the expression for the dot product of two commuting tensor operators as

$$
\begin{aligned}
V_{c c^{\prime}}^{\mathcal{J}}(r) & =\sum_{L S J}(-1)^{j_{c^{\prime}}+I_{c}+\mathcal{J}}\left\{\begin{array}{lll}
\mathcal{J} & I_{c} & j_{c} \\
J & j_{c^{\prime}} & I_{c^{\prime}}
\end{array}\right\} \\
& \times\left(\beta_{c}\left(l_{c} s_{c}\right) j_{c}\left\|\left[Y_{L}(\hat{\mathbf{r}}) \times Q_{S}^{(p)}\right]_{J}\right\| \beta_{c^{\prime}}\left(l_{c^{\prime}} s_{c^{\prime}}\right) j_{c^{\prime}}\right)\left(\alpha_{c} I_{c}\left\|Q_{J}^{(t)}\right\| \alpha_{c^{\prime}} I_{c^{\prime}}\right) .
\end{aligned}
$$


The first matrix element can be factorized as

$$
\begin{aligned}
\left(\beta_{c}\left(l_{c} s_{c}\right) j_{c} \|\left[Y_{L}\right.\right. & \left.\left.\times Q_{S}^{(p)}\right]_{J} \| \beta_{c^{\prime}}\left(l_{c^{\prime}} s_{c^{\prime}}\right) j_{c^{\prime}}\right) \\
& =\hat{j_{c}} \hat{j_{c^{\prime}}} \hat{J}\left\{\begin{array}{lll}
l_{c} & l_{c^{\prime}} & L \\
s_{c} & s_{c^{\prime}} & S \\
j_{c} & j_{c^{\prime}} & J
\end{array}\right\}\left(l_{c}\left\|Y_{L}\right\| l_{c^{\prime}}\right)\left(\beta_{c} s_{c}\left\|Q_{S}^{(p)}\right\| \beta_{c^{\prime}} s_{c^{\prime}}\right)
\end{aligned}
$$

and the orbital matrix element is evaluated as

$$
\left(l_{c} \| Y_{L}|| l_{c^{\prime}}\right)=i^{l_{c^{\prime}}-l_{c}}(-1)^{l_{c}} \frac{1}{\sqrt{4 \pi}} \hat{l_{c}} \hat{l_{c^{\prime}}} \hat{L}\left(\begin{array}{ccc}
l_{c} & L & l_{c^{\prime}} \\
0 & 0 & 0
\end{array}\right),
$$

where we have now made the $i^{l}$ factors in each channel explicit (see Eq. 5.3). Eq. 6.9 then becomes

$$
\begin{aligned}
\left(\beta_{c}\left(l_{c} s_{c}\right) j_{c} \|\left[Y_{L}\right.\right. & \left.\left.\times Q_{S}^{(p)}\right]_{J} \| \beta_{c^{\prime}}\left(l_{c^{\prime}} s_{c^{\prime}}\right) j_{c^{\prime}}\right)=i^{l_{c^{\prime}}-l_{c}} \frac{1}{\sqrt{4 \pi}}(-1)^{l_{c}} \hat{l_{c}} \hat{l_{c^{\prime}}} \hat{j_{c}} \hat{j_{c^{\prime}}} \hat{L} \hat{J} \\
& \times\left(\begin{array}{ccc}
l_{c} & l_{c^{\prime}} & L \\
0 & 0 & 0
\end{array}\right)\left\{\begin{array}{ccc}
l_{c} & l_{c^{\prime}} & L \\
s_{c} & s_{c^{\prime}} & S \\
j_{c} & j_{c^{\prime}} & J
\end{array}\right\}\left(\beta_{c} s_{c}\left\|Q_{S}^{(p)}\right\| \beta_{c^{\prime}} s_{c^{\prime}}\right) .
\end{aligned}
$$

We put this last expression into Eq. 6.8 to get the complete expression for the coupling potential,

$$
V_{c c^{\prime}}^{\mathcal{J}}(r)=i^{l^{c^{\prime}}-l_{c}} \sum_{L S J} F_{L S J}^{\mathcal{J}}\left(l_{c} s_{c} j_{c} I_{c} ; l_{c^{\prime}} s_{c^{\prime}} j_{c^{\prime}} I_{c^{\prime}}\right)\left(\beta_{c} s_{c}\left\|Q_{S}^{(p)}\right\| \beta_{c^{\prime}} s_{c^{\prime}}\right)\left(\alpha_{c} I_{c}\left\|Q_{J}^{(t)}\right\| \alpha_{c^{\prime}} I_{c^{\prime}}\right),
$$

where we have defined an angular momentum coupling coefficient

$$
\begin{aligned}
& F_{L S J}^{\mathcal{J}}\left(l_{c} s_{c} j_{c} I_{c} ; l_{c^{\prime}} s_{c^{\prime}} j_{c^{\prime}} I_{c^{\prime}}\right)=\frac{1}{\sqrt{4 \pi}}(-1)^{j_{c^{\prime}}+I_{c}+\mathcal{J}+l_{c}} \hat{l_{c}} \hat{l_{c^{\prime}}} \hat{j_{c}} \hat{j_{c^{\prime}}} \hat{L} \hat{J} \\
& \times\left\{\begin{array}{ccc}
j_{c} & j_{c^{\prime}} & J \\
I_{c^{\prime}} & I_{c} & \mathcal{J}
\end{array}\right\}\left(\begin{array}{ccc}
l_{c} & l_{c^{\prime}} & L \\
0 & 0 & 0
\end{array}\right)\left\{\begin{array}{ccc}
l_{c} & l_{c^{\prime}} & L \\
s_{c} & s_{c^{\prime}} & S \\
j_{c} & j_{c^{\prime}} & J
\end{array}\right\} .
\end{aligned}
$$

\subsubsection{Coupling Potentials in FRESCO}

We are interested in defining what must be supplied to Fresco when numerical form factors are used to describe the coupling potentials. We refer to Appendix V: KIND=1 Spin Transfer Couplings in the FRESCO input manual [3], which contains additional information on the meaning of the angular momentum coefficients. In comparing with the present results, we use Table 6.1 to translate symbols. The documentation for Fresco in Computer Physics Reports [4] indicates that the $i^{l_{c^{\prime}}-l_{c}}$ phase factor is not included in the potential (see Eq. 30 in Ref. [4]). We thus make the identification

$$
V_{c c^{\prime}}^{\mathcal{J}}(r)=i^{l^{\prime}-l_{c}} \sum_{L S J} V_{L S J, c c^{\prime}}^{\mathfrak{d}, \mathrm{FRESCO}}(r)
$$




\begin{tabular}{|l|cccccccccccc|}
\hline here & $l_{c}$ & $s_{c}$ & $j_{c}$ & $I_{c}$ & $l_{c^{\prime}}$ & $s_{c^{\prime}}$ & $j_{c^{\prime}}$ & $I_{c^{\prime}}$ & $L$ & $S$ & $J$ & $\mathcal{\partial}$ \\
\hline FRESCO & $L$ & $I_{p}$ & $J$ & $I_{t}$ & $L^{\prime}$ & $I_{p}^{\prime}$ & $J^{\prime}$ & $I_{t}^{\prime}$ & $\ell$ & $s_{p}$ & $s_{t}$ & $J_{T}$ \\
\hline
\end{tabular}

Table 6.1: Correspondence between variables in this report and in the Fresco description.

where after translation of the symbols the expression for the coupling potential in the input manual can be written as

$$
\begin{aligned}
& V_{L S J, c c^{\prime}}^{\mathcal{J}, \mathrm{FRESCO}}(r)= \\
& \quad \times(-1)^{J+\mathcal{J}+j_{c^{\prime}}+I_{c}}\left\{\begin{array}{ccc}
j_{c^{\prime}} & I_{c^{\prime}} & \mathcal{J} \\
I_{c} & j_{c} & J
\end{array}\right\} \hat{j_{c}} \hat{j_{c^{\prime}}}\left\{\begin{array}{ccc}
l_{c^{\prime}} & s_{c^{\prime}} & j_{c^{\prime}} \\
L & S & J \\
l_{c} & s_{c} & j_{c}
\end{array}\right\} \frac{1}{\sqrt{4 \pi}} \hat{L} \hat{l_{c^{\prime}}}\left(l_{c^{\prime}} 0 L 0 \mid l_{c} 0\right) \\
& \quad \times K(r),
\end{aligned}
$$

where we have made corrections to Eq. (4) in Appendix V of Ref. [3] after consultation with the author. According to the input manual, when using couplings with KIND=1 and IP3 $=0$, the program supplies the second line automatically and the user must supply the factor $K(r)$.

The task now is to identify $K(r)$ so that Eq. 6.14 is satisfied. We convert the ClebschGordan coefficient in the above expression to a 3 -j symbol by

$$
\left(l_{c^{\prime}} 0 L 0 \mid l_{c} 0\right)=(-1)^{l_{c^{\prime}}-L} \sqrt{2 l_{c}+1}\left(\begin{array}{ccc}
l_{c^{\prime}} & L & l_{c} \\
0 & 0 & 0
\end{array}\right)
$$

The 3-j, 6-j, and 9-j symbols appearing in Eq. 6.13 for the geometric factor $F_{L S J}^{\mathcal{J}}$ and the corresponding symbols in the two expressions above are identical, as can be seen by applying the appropriate symmetry relations. Then by comparing the geometrical coefficient in Eq. 6.15 with $F_{L S J}^{\mathcal{J}}$ we can write

$$
V_{L S J, c c^{\prime}}^{\mathcal{J}, \mathrm{FrESCO}}(r)=F_{L S J}^{\mathcal{J}}\left(l_{c} s_{c} j_{c} I_{c} ; l_{c^{\prime}} s_{c^{\prime}} j_{c^{\prime}} I_{c^{\prime}}\right)(-1)^{J} \frac{1}{\sqrt{2 J+1}} K(r)
$$

and by comparing with Eq. 6.12 we identify the factor the user must supply as

$$
K(r)=(-1)^{J} \sqrt{2 J+1}\left(\beta_{c} s_{c}\left\|Q_{S}^{(p)}\right\| \beta_{c^{\prime}} s_{c^{\prime}}\right)\left(\alpha_{c} I_{c}\left\|Q_{J}^{(t)}\right\| \alpha_{c^{\prime}} I_{c^{\prime}}\right) .
$$

The factor $(-1)^{J} \sqrt{2 J+1}$ simply corresponds to the difference between the dot-product and vector-coupled forms for the rotationally-invariant operator.

Some questions relevant to this method for the input of externally-supplied potentials in Fresco are

- The manual (Ref. [3]) indicates that the radial shapes for the form factors have to be read in using Cards 16.7. Card 16.6 is intended. Following this card, the function $K(r)$ is read in using format $6 \mathrm{f} 12.0$, not free format. A blank line should not be placed after each form factor, but rather at the end of the file when there are several form factors or other items in FILE4. 
- According to the author, the form factor below the radius for the first externallysupplied point is given the value of the form factor at the first point. The form factor above the last point is extrapolated as $1 / r$.

We can now relate $K(r)$ to the coupling potentials $U_{L S J}^{c c^{\prime}}(r)$ for inelastic scattering and charge exchange in Sec. 5.2. To do this we equate Eq. 5.35 for $V_{c c^{\prime}}^{\mathcal{J}}(r)$ to Eq. 6.14, and insert the expression Eq. 6.17 for $V_{L S J, c c^{\prime}}^{\mathcal{J}, \text { FreSCO}}(r)$. The result is

$$
K(r)=(-1)^{L+S} \sqrt{(2 J+1)\left(2 I_{c}+1\right)}\left(s\left\|\mathcal{S}_{S}\right\| s\right) U_{L S J}^{c c^{\prime}}(r),
$$

where in this case the projectile is unchanged by the scattering, so that we can set $s_{c}=s_{c^{\prime}}=$ $s$; specific values of the matrix element for spin- 0 and spin- $\frac{1}{2}$ projectiles are

$$
\begin{aligned}
\left(s\left\|\mathcal{S}_{S}\right\| s\right) & =1 & & (\text { projectile spin } s=0) \\
& =\sqrt{2(2 S+1)} & & \left(\text { projectile spin } s=\frac{1}{2}\right) .
\end{aligned}
$$

Note that when the coupled-channels calculation is solved to first order as a distorted-wave approximation calculation, $c^{\prime}$ is the initial channel and $c$ is the final channel; this convention is opposite to that in Chapter 4 where the final channel quantum numbers are primed. If the potential is available in momentum space as $U_{L S J}^{c c^{\prime}}(q)$, its value $U_{L S J}^{c c^{\prime}}(r)$ in coordinate space is calculated by the inverse Fourier transformation of Eq. C.9 or C.11.

\subsubsection{Coupling Potentials from Tamura}

Tamura's review article [5] treats only couplings without spin transfer $(S=0)$, and assumes the coupling does not change the projectile spin $\left(s_{c}=s_{c^{\prime}}=s\right)$.

We relate Tamura's $A$ coefficient (Eq. 28 in Ref. [5]) to the geometric coefficient $F_{L S J}^{\mathcal{J}}$ of our Eq. 6.13. We note a misprint in Tamura's equation - the factors $l l^{\prime}$ immediately preceding the Clebsch-Gordan coefficient should have hats. With this correction, we find in our notation the correspondence

$$
A\left(l_{c} j_{c} I_{c}, l_{c^{\prime}} j_{c^{\prime}} I_{c^{\prime}}, J \mathcal{J} s\right)=i^{l_{c^{\prime}}-l_{c}} \sqrt{2 s+1} F_{J 0 J}^{\mathcal{J}}\left(l_{c} s j_{c} I_{c} ; l_{c^{\prime}} s j_{c^{\prime}} I_{c^{\prime}}\right) .
$$

The projectile reduced matrix element is

$$
\left(\beta_{c} s_{c}\left\|Q_{S}^{(p)}\right\| \beta_{c^{\prime}} s_{c^{\prime}}\right) \rightarrow\left(s\left\|(1)_{0}^{(p)}\right\| s\right)=1 / \sqrt{2 s+1} .
$$

and thus we can write the coupling potential as

$$
V_{c c^{\prime}}^{\mathcal{J}}(r)=i^{l_{c^{\prime}}-l_{c}} \sum_{J} F_{J 0 J}^{\mathcal{J}}\left(l_{c} s j_{c} I_{c} ; l_{c^{\prime}} s j_{c^{\prime}} I_{c^{\prime}}\right)\left(\alpha_{c} I_{c}\left\|Q_{J}^{(t)}\right\| \alpha_{c^{\prime}} I_{c^{\prime}}\right),
$$

where the relation between the target matrix element and the quantities in Tamura's article is

$$
\left(\alpha_{c} I_{c}\left\|Q_{J}^{(t)}\right\| \alpha_{c^{\prime}} I_{c^{\prime}}\right)=\sum_{n} v_{J}^{(n)}(r)\left\langle I_{c}\left\|Q_{J}^{(t)(n)}\right\| I_{c^{\prime}}\right\rangle
$$

where the symbol $t$ as used here indicates that the matrix element is in the target Hilbert space; unfortunately Tamura uses the same index to distinguish different tensors of the same rank. We therefore use $n$ for this purpose. The above results should be sufficient to allow translation of the expressions in Tamura's paper into equivalent ones for use in other codes. 


\subsection{Implementation of Folding Procedure}

\subsubsection{The ALLWRLD Code}

The densities in the ALLWRLD code of Carr, Kelly, and Petrovich [6] are calculated in a harmonic-oscillator basis using the expressions in Sec. 3.1, Eqs. 3.17-3.19 and Sec. B.2, with the following exceptions and additional comments:

- The factor $i^{l}$ in Eq. B.21 is omitted, and consequently the factor $i^{l_{1}-l_{2}}$ in Eq. B.22 is absent.

- The phases of the harmonic oscillator wave functions are chosen to be positive near the origin.

- The factor $(4 \pi)^{-1}$ in the definition of the coordinate-space radial density (Eq. 3.11) is omitted. As a result, the coordinate-space radial densities are larger than described in Sec. 3.1 by a factor $4 \pi$, but the momentum-space radial densities are the same as in Sec. 3.1.

Table 6.2 shows the correspondence between the momentum-space densities in Sec. 3.1 and their names in the ALLWRLD output for inelastic scattering.

\begin{tabular}{|c|c|}
\hline \hline \multicolumn{2}{|c|}{ Natural parity, $L=J$} \\
\hline$\rho_{L S J}$ & ALLWRLD \\
\hline \hline$\rho_{J 0 J}$ & rqj \\
$\rho_{J 1 J}$ & rqsjj \\
\hline \hline \multicolumn{2}{|c|}{ Unnatural parity, $L=J \pm 1$} \\
\hline$\rho_{L S J}$ & ALLWRLD \\
\hline \hline$\rho_{J-11 J}$ & rqsjll \\
$\rho_{J+11 J}$ & rqsjlu \\
\hline
\end{tabular}

Table 6.2: Correspondence between densities in Sec. 3.1 and notation in ALLWRLD output for q-space densities for inelastic scattering. See text regarding normalization and phase conventions.

\subsubsection{The Author's Code, PWA}

The current implementation of PWA calculates the matter and spin densities as described in Sec. 3.1, Eqs. 3.17-3.19. The single-particle reduced matrix element in Eq. 3.17 is evaluated as in Sec. B.2, except that the factors $i^{l}$ and $i^{l_{1}-l_{2}}$ in Eqs. B.21 and B.22 are omitted. The single-particle radial wave functions are defined so that they are always positive near the origin. This leads to $Z$ amplitudes defined in a basis with exactly the same single-particle phases as in the ALLWRLD program.

In comparisons with ALLWRLD, tests using harmonic-oscillator wave functions yield identical results for the momentum-space radial densities. For the coordinate-space radial densities, the values from ALLWRLD are larger than from the author's code by a factor of $4 \pi$. 
This results from the choice made in Sec. 3.1 to include a factor of $(4 \pi)^{-1}$ along with the radial delta function in the definition of the r-space radial density (Eq. 3.11). This choice was made to ensure that the $\mathrm{r}$ - and q-space radial densities are related by the expressions for Fourier transforms in Appendix C.2 without an extra numerical factor. 


\section{Chapter 7}

\section{Inelastic Scattering Using RPA Amplitudes}

\subsection{Introduction}

To calculate scattering to excited states whose structure is described by the random phase approximation (RPA), we need to relate the amplitudes obtained from the RPA calculation (usually called $X$ and $Y$ coefficients) to the spectroscopic amplitudes $Z$ as described in Section B.1. These $Z$ coefficients can then be used either directly in an inelastic-scattering code that uses them as input, or they may combined with particle and hole radial wave functions to generate a transition density that can be folded with an effective interaction to generate a transition potential on a radial mesh.

We illustrate these procedures here using the techniques, definitions and phase conventions embodied in the Hartree-Fock and RPA codes written at Bruyères-le-Châtel. A version of these codes is available at Livermore, and they are being used to generate information on angular momentum and parity transfer in inelastic scattering reactions to highly excited states. The codes were supplied by Marc DuPuis, who used (and extended) them for closelyrelated work in his thesis project. A full account of the Hartree-Fock theory and the RPA can be found in texts such as that of Ring and Schuck [7]; the thesis of Marc DuPuis [8] (in French) contains a useful summary of the theory as embodied in the Bruyères-le-Châtel codes.

We consider states $|N J M\rangle$ based on the exact (i.e. correlated) ground state $|0\rangle$. We assume a $0^{+}$ground state, and $N$ distinguishes the different excited states of given angular momentum $J$ and parity; we henceforth suppress the parity quantum number of the excited states.

The state vectors used to describe the Hartree-Fock basis are defined with the same conventions as in Eq. B.21; namely

$$
|n l s j m t\rangle=\frac{\phi_{n l j t}(r)}{r} i^{l}\left[Y_{l}(\hat{\mathbf{r}}) \times \chi_{\frac{1}{2}}\right]_{j m} \chi_{\frac{1}{2} t}^{i s o},
$$

where the radial wave functions $\phi_{n l j t}(r)$ are described as an expansion in a harmonic-oscillator basis. With these state vectors we associate the creation and annihilation operators $a_{n l s j m t}^{\dagger}$ 
and $a_{n l s j m t}$. It is convenient to define a modified annihilation operator,

$$
\tilde{a}_{n l s j m t}=(-1)^{j-m} a_{n l s j m t},
$$

which is a spherical tensor of rank $j$ and projection $-m$.

We now define particle $(p)$ and hole $(h)$ states corresponding to orbitals above or below the Fermi surface, schematically indicated by $p>F$ and $p<F$, respectively. The symbol $p$ represents all quantum numbers except the magnetic projection; i.e. $p \equiv\left\{n_{p} l_{p} \frac{1}{2} j_{p} t_{p}\right\}$. The same definition applies to the hole states; replace $p$ by $h$.

With the above definitions we define an operator that creates a particle-hole pair coupled to angular momentum $J$ and projection $M$,

$$
\mathcal{A}_{J M}^{\dagger}(p, \tilde{h})=\sum_{m_{p} m_{h}}\left(j_{p} m_{p} j_{h}-m_{h} \mid J M\right) a_{p m_{p}}^{\dagger} \tilde{a}_{h m_{h}} .
$$

This definition is identical to that of Eq. B.4 used to define the $Z$ coefficient in Eq. B.7 in Section B.1. From the Hermitian conjugate of this, we define another operator that destroys a particle-hole pair,

$$
\mathcal{A}_{J \bar{M}}(p, \tilde{h})=(-1)^{J-M} \mathcal{A}_{J-M}(p, \tilde{h}) .
$$

Both of the above expressions are spherical tensors of rank $J$ and projection $M$. The last expression can be written in terms of the particle and hole operators as

$$
\mathcal{A}_{J \bar{M}}(p, \tilde{h})=(-1)^{J-j_{p}+j_{h}} \sum_{m_{p} m_{h}}\left(j_{h} m_{h} j_{p}-m_{p} \mid J M\right) a_{h m_{h}}^{\dagger} \tilde{a}_{p m_{p}} .
$$

This also is in the form of the $A$ operator of Eq. B.4, except for the sign factor preceding the summation.

We define a boson operator $\Theta_{N J M}^{\dagger}$ that creates the RPA state $|N J M\rangle$ when applied to the correlated ground state $|0\rangle$, which is the vacuum for the RPA excitations. We also define the time-reversed destruction operator $\Theta_{N J \bar{M}}=(-1)^{J-M} \Theta_{N J-M}$. The relation between the particle-hole operators and the boson operators is

$$
\begin{aligned}
& \mathcal{A}_{J M}^{\dagger}(p, \tilde{h})=\sum_{N} X_{p h}^{N J} \Theta_{N J M}^{\dagger}+Y_{p h}^{N J} \Theta_{N J \bar{M}} \\
& \mathcal{A}_{J \bar{M}}(p, \tilde{h})=\sum_{N} X_{p h}^{N J} \Theta_{N J \bar{M}}+Y_{p h}^{N J} \Theta_{N J M}^{\dagger},
\end{aligned}
$$

where $X_{p h}^{N J}$ and $Y_{p h}^{N J}$ are obtained as the solutions of the RPA equations. By taking matrix elements of these equations between the excited and ground states, it follows that

$$
\begin{aligned}
\left\langle N J M\left|\mathcal{A}_{J M}^{\dagger}(p, \tilde{h})\right| 0\right\rangle & =X_{p h}^{N J} \\
\left\langle N J M\left|\mathcal{A}_{J M}(p, \tilde{h})\right| 0\right\rangle & =Y_{p h}^{N J} .
\end{aligned}
$$

The $X$ and $Y$ coefficients are real. Their well-known normalization and orthogonality relation is

$$
\sum_{\substack{p>F \\ h<F}} X_{p h}^{N J} X_{p h}^{N^{\prime} J}-Y_{p h}^{N J} Y_{p h}^{N^{\prime} J}=\delta_{N N^{\prime}}
$$




\section{2 $Z$ Coefficients and Transition Density in the RPA}

From Eq. B.7 the spectroscopic amplitudes ( $Z$ coefficients) relevant to the problem at hand are defined by

$$
\begin{aligned}
Z_{t t}^{N J}\left(\alpha_{2} j_{2}, \alpha_{1} j_{1}\right) & =\frac{1}{\sqrt{2 J+1}}\left(N J\left\|A_{t t}^{J}\left(\alpha_{2} j_{2}, \alpha_{1} j_{1}\right)\right\| 0\right) \\
& =\left\langle N J M\left|A_{t t}^{J M}\left(\alpha_{2} j_{2}, \alpha_{1} j_{1}\right)\right| 0\right\rangle
\end{aligned}
$$

where the initial (ground) state has spin 0 . We need to keep only $t_{1}=t_{2}=t$ (where $t= \pm \frac{1}{2}$ ) since the RPA excitations do not change the nuclear charge. Also, we have chosen to add the superscript $N$ in the symbol $Z_{t t}^{N J}$ to specify the excited state completely.

The RPA amplitudes of Eqs. 7.8,7.9 involve only the creation or destruction of a particlehole pair; there are no particle-particle or hole-hole terms. We thus need only the coefficients

$$
\begin{aligned}
& Z_{t t}^{N J}(p, h)=\left\langle N J M\left|A_{t t}^{J M}(p, h)\right| 0\right\rangle \\
& Z_{t t}^{N J}(h, p)=\left\langle N J M\left|A_{t t}^{J M}(h, p)\right| 0\right\rangle
\end{aligned}
$$

where $p$ represents the particle Hartree-Fock states $(p>F)$, and $h$ the hole states $(h<F)$. From the definitions of the $A_{t t}^{J M}$ operator of Eq. B.4 and those of the $\mathcal{A}_{J M}^{\dagger}$ and $\mathcal{A}_{J \bar{M}}$ operators of Eqs. 7.3,7.5 we see that

$$
\begin{aligned}
& \mathcal{A}_{J M}^{\dagger}(p, \tilde{h})=A_{t t}^{J M}(p, h) \\
& \mathcal{A}_{J M}(p, \tilde{h})=(-1)^{J-j_{p}+j_{h}} A_{t t}^{J M}(h, p) .
\end{aligned}
$$

Inserting the values for the $A_{t t}^{J M}$ operators from these expressions into Eqs. 7.13,7.14 and using the expressions for the $X_{p h}^{N J}$ and $Y_{p h}^{N J}$ coefficients of Eqs. 7.8,7.9 yields the results

$$
\begin{aligned}
& Z_{t t}^{N J}(p, h)=X_{p h}^{N J} \\
& Z_{t t}^{N J}(h, p)=(-1)^{J-j_{p}+j_{h}} Y_{p h}^{N J} .
\end{aligned}
$$

These, together with $Z_{t t}^{N J}\left(p, p^{\prime}\right)=Z_{t t}^{N J}\left(h, h^{\prime}\right)=0$, fully specify the specify the spectroscopic amplitudes required to calculate the densities, as well as a wide class of other one-body operators.

From Eqs. 3.16-3.19 the coordinate-space neutron (or proton) density is

$$
\begin{aligned}
\rho_{L S J}^{n(p)}\left(r_{t}\right)= & \sum_{\substack{n_{1} l_{1} j_{1} \\
n_{2} l_{2} j_{2}}} \frac{1}{\sqrt{2 J+1}} Z_{t t}^{N J}\left(n_{2} l_{2} j_{2}, n_{1} l_{1} j_{1}\right) \\
& \quad \times\left(n_{2} l_{2} j_{2} t\left\|\frac{1}{4 \pi} \frac{\delta\left(r_{t}-r\right)}{r_{t} r}\left[Y_{L}\left(\hat{\mathbf{r}}_{t}\right) \times \mathcal{S}_{S}\right]_{J}\right\| n_{1} l_{1} j_{1} t\right),
\end{aligned}
$$

where $r_{t}$ refers to the radial coordinate of the density for the collection of nucleons in the target nucleus; otherwise the quantity $t$ takes the value $+\frac{1}{2}$ or $-\frac{1}{2}$ to indicate neutron or proton single-particle states, respectively. Using the Hartree-Fock basis functions of Eq. 7.1 
we can write the density as

$$
\begin{aligned}
i^{L} \rho_{L S J}^{n(p)}\left(r_{t}\right)=\frac{1}{4 \pi} & \frac{1}{\sqrt{2 J+1}} \sum_{\substack{n_{1} l_{1} j_{1} \\
n_{2} l_{2} j_{2}}} Z_{t t}^{N J}\left(n_{2} l_{2} j_{2}, n_{1} l_{1} j_{1}\right) \\
& \times i^{l_{1}-l_{2}+L}\left(l_{2} \frac{1}{2} j_{2}\left\|\left[Y_{L}\left(\hat{\mathbf{r}}_{t}\right) \times \mathcal{S}_{S}\right]_{J}\right\| l_{1} \frac{1}{2} j_{1}\right) \frac{\phi_{n_{2} l_{2} j_{2} t}\left(r_{t}\right)}{r_{t}} \frac{\phi_{n_{1} l_{1} j_{1} t}\left(r_{t}\right)}{r_{t}},
\end{aligned}
$$

where we have multiplied the density by the factor $i^{L}$. This ensures that the right-hand side of the expression is real, since we are working in a representation in which the radial functions are real and the factor $i^{l_{1}-l_{2}+L}$ is real. This last observation follows by noting that $l_{1}-l_{2}+L$ is even, as a consequence of the parity selection rule in the 3 -j symbol in Eq. B.26.

We now take advantage of the fact that the state $n_{1} l_{1} j_{1}$ must be a particle state (which we represent by $p$ ) and $n_{2} l_{2} j_{2}$ a hole state (represented by $h$ ), or vice versa. The last expression can be written as

$$
\begin{aligned}
& i^{L} \rho_{L S J}^{n(p)}\left(r_{t}\right)=\frac{1}{4 \pi} \frac{1}{\sqrt{2 J+1}} \sum_{\substack{p>F \\
h<F}} \\
& \quad\left[Z_{t t}^{N J}(p, h) i^{l_{h}-l_{p}+L}\left(l_{p} \frac{1}{2} j_{p}\left\|\left[Y_{L}\left(\hat{\mathbf{r}}_{t}\right) \times \mathcal{S}_{S}\right]_{J}\right\| l_{h} \frac{1}{2} j_{h}\right)\right. \\
& \left.+Z_{t t}^{N J}(h, p) i^{l_{p}-l_{h}+L}\left(l_{h} \frac{1}{2} j_{h}\left\|\left[Y_{L}\left(\hat{\mathbf{r}}_{t}\right) \times \mathcal{S}_{S}\right]_{J}\right\| l_{p} \frac{1}{2} j_{p}\right)\right] \\
& \quad \times \frac{\phi_{p}\left(r_{t}\right)}{r_{t}} \frac{\phi_{h}\left(r_{t}\right)}{r_{t}} .
\end{aligned}
$$

We insert the values of Eqs. 7.17 and 7.18 for the $Z$ coefficients into this expression, and use the symmetry relation of Eq. B.27 to interchange the order of the particle and hole state in the second reduced matrix element. The result is

$$
\begin{aligned}
i^{L} \rho_{L S J}^{n(p)}\left(r_{t}\right)= & \frac{1}{4 \pi} \frac{1}{\sqrt{2 J+1}} \sum_{\substack{p>F \\
h<F}}^{\prime}\left[X_{p h}^{N J}+(-1)^{S} Y_{p h}^{N J}\right] \\
& \times i^{l_{h}-l_{p}+L}\left(l_{p} \frac{1}{2} j_{p}\left\|\left[Y_{L}\left(\hat{\mathbf{r}}_{t}\right) \times \mathcal{S}_{S}\right]_{J}\right\| l_{h} \frac{1}{2} j_{h}\right) \frac{\phi_{p}\left(r_{t}\right)}{r_{t}} \frac{\phi_{h}\left(r_{t}\right)}{r_{t}} .
\end{aligned}
$$

This is now in a form suitable for numerical computation, using the expressions for the reduced matrix element of the single-particle operator in Section B.2. For neutron densities the sum is over only neutron particle-hole pairs, while for proton densities it is over only proton particle-hole pairs; this condition is indicated by the prime on the summation sign. As a special case, the ordinary (i.e. $S=0$ ) density is

$$
\begin{aligned}
i^{J} \rho_{J 0 J}^{n(p)}\left(r_{t}\right) & =\left(\frac{1}{4 \pi}\right)^{\frac{3}{2}} \sum_{\substack{p>F \\
h<F}}^{\prime}\left(X_{p h}^{N J}+Y_{p h}^{N J}\right) \\
& \times i^{l_{h}-l_{p}-J}(-1)^{j_{h}-\frac{1}{2}} \hat{j}_{p} \hat{j}_{h}\left(\begin{array}{ccc}
j_{p} & J & j_{h} \\
-\frac{1}{2} & 0 & \frac{1}{2}
\end{array}\right) \frac{\phi_{p}\left(r_{t}\right)}{r_{t}} \frac{\phi_{h}\left(r_{t}\right)}{r_{t}} .
\end{aligned}
$$

We find that calculations of Eq. 7.22 for $S=0$, if multiplied by $4 \pi$, agree with the transition densities printed out by the Bruyères-le-Châtel RPA code we have available. This factor of 
$4 \pi$ is a consequence of the particular definition of the normalization of the transition density chosen in the present work; see the discussion immediately following Eq. 3.14. There is an expression similar to Eq. 7.23 in Marc DuPuis's thesis, which has a slightly different phase factor; we believe the result of Eq. 7.23 is correct. 


\section{Acknowledgments}

This work was performed under the auspices of the U.S. Department of Energy by the University of California, Lawrence Livermore National Laboratory under contract No. W-7405Eng-48. Partial funding was provided by the Laboratory Directed Research and Development Program at LLNL under project 04-ERD-057. The author wishes to gratefully acknowledge many years of fruitful collaboration with the late Prof. Fred Petrovich on projects related to the topics in this report. 


\section{Appendix A}

\section{Angular-Momentum Conventions and Other Definitions}

\section{A.1 General Remarks}

- The conventions of Edmonds [2] are used for angular momentum quantities (rotation matrices, reduced matrix elements, 3-, 6-, and 9-j symbols, etc.

- A hat on a vector indicates that it is a unit vector; e.g. $\hat{\mathbf{x}}$ is a unit vector in the direction of $\mathbf{x}$.

- A hat on a symbol representing an angular momentum has the following significance:

$$
\hat{J} \equiv \sqrt{2 J+1} \text {. }
$$

- We frequently use the following notation to indicate vector coupling, as well as the order of coupling:

$$
\left[A_{J_{1}} \times B_{J_{2}}\right]_{J_{3} M_{3}}=\sum_{M_{1} M_{2}}\left(J_{1} M_{1} J_{2} M_{2} \mid J_{3} M_{3}\right) A_{J_{1} M_{1}} B_{J_{2} M_{2}} .
$$

- The dot product of two tensors of the same rank, as defined by Edmonds [2], Eq. 5.2.4, p. 72 is

$$
\begin{aligned}
A_{J} \cdot B_{J} & =\sum_{M} A_{J M}(-1)^{M} B_{J-M} \\
& =(-1)^{J} \sqrt{2 J+1}\left[A_{J} \times B_{J}\right]_{00} .
\end{aligned}
$$

- The factor $\hbar$ is normally omitted in writing angular momentum operators.

\section{A.2 Definitions of $\bar{Z}$ and $\bar{Z}_{1}$ Coefficients}

The $\bar{Z}$ and $\bar{Z}_{1}$ coefficients are combinations of $3-\mathrm{j}$ and 6 -j symbols that frequently appear for absorption or emission of particles and gamma rays, respectively. There are variations in definition among different authors, and so we show here the definitions we use. 
The following definition of $\bar{Z}$ is exactly equivalent to that in Lane and Thomas [9], Eq. 2.8a on page 292, and also to that used in [10]:

$$
\begin{aligned}
\bar{Z}\left(l j l^{\prime} j^{\prime} ; j_{1} k\right)= & (-1)^{j+j^{\prime}} \sqrt{(2 l+1)\left(2 l^{\prime}+1\right)(2 j+1)\left(2 j^{\prime}+1\right)(2 k+1)} \\
& \times\left(\begin{array}{ccc}
l & l^{\prime} & k \\
0 & 0 & 0
\end{array}\right)\left\{\begin{array}{ccc}
l & j & j_{1} \\
j^{\prime} & l^{\prime} & k
\end{array}\right\} .
\end{aligned}
$$

The following definition of $\bar{Z}_{1}$ is the one used in the radiative capture calculations of Parker et al. [10]:

$$
\begin{aligned}
\bar{Z}_{1}^{\sigma \sigma^{\prime}}\left(L J L^{\prime} J^{\prime} ; J_{1} k\right)= & (-1)^{L+J+L^{\prime}+J^{\prime}+k} \\
& \times \sqrt{(2 L+1)\left(2 L^{\prime}+1\right)(2 J+1)\left(2 J^{\prime}+1\right)(2 k+1)} \\
& \times(-1)\left[\frac{1+(-1)^{\sigma+\sigma^{\prime}+L+L^{\prime}+k}}{2}\right]\left(\begin{array}{ccc}
L & L^{\prime} & k \\
-1 & 1 & 0
\end{array}\right)\left\{\begin{array}{ccc}
L & J & J_{1} \\
J^{\prime} & L^{\prime} & k
\end{array}\right\} .
\end{aligned}
$$

Here $L, \sigma$ (and $L^{\prime}, \sigma^{\prime}$ ) represent the gamma multipolarity; $\sigma=0$ for electric multipoles and $\sigma=1$ for magnetic.

\section{A.3 Special Relations among 3-j, 6-j, and 9-j Symbols}

Several special relations among angular momentum coefficients are useful in simplifying or speeding up calculations. Care should be exercised in using the following, since the angular momentum selection rules are not always automatically enforced.

The following connection between 3 -j and 6 -j symbols is equivalent to that in deShalit and Talmi [11] (p. 519):

$$
\left(\begin{array}{ccc}
j_{2} & J & j_{1} \\
-\frac{1}{2} & 0 & \frac{1}{2}
\end{array}\right)=-\sqrt{\left(2 l_{1}+1\right)\left(2 l_{2}+1\right)}\left(\begin{array}{ccc}
l_{2} & l_{1} & J \\
0 & 0 & 0
\end{array}\right)\left\{\begin{array}{lll}
j_{1} & j_{2} & J \\
l_{2} & l_{1} & \frac{1}{2}
\end{array}\right\} .
$$

The following relation connects 9-j symbols:

$$
\left\{\begin{array}{ccc}
s & s & 1 \\
l_{1} & l_{2} & J \\
j_{1} & j_{2} & J
\end{array}\right\}=\frac{l_{1}\left(l_{1}+1\right)-l_{2}\left(l_{2}+1\right)-j_{1}\left(j_{1}+1\right)+j_{2}\left(j_{2}+1\right)}{2 \sqrt{s(s+1) J(J+1)}}\left\{\begin{array}{ccc}
s & s & 0 \\
l_{1} & l_{2} & J \\
j_{1} & j_{2} & J
\end{array}\right\} .
$$

This relation is stated incorrectly in Rotenberg et al. [12] (Eq. 3.24, p. 24).

The last expression may be simplified by using the expression for a $9-\mathrm{j}$ symbol with a zero argument. The result is

$$
\begin{aligned}
& \left\{\begin{array}{ccc}
s & s & 1 \\
l_{1} & l_{2} & J \\
j_{1} & j_{2} & J
\end{array}\right\}=(-1)^{j_{1}+s+l_{2}+J} \\
& \quad \times \frac{l_{1}\left(l_{1}+1\right)-l_{2}\left(l_{2}+1\right)-j_{1}\left(j_{1}+1\right)+j_{2}\left(j_{2}+1\right)}{2 \sqrt{s(s+1)(2 s+1) J(J+1)(2 J+1)}}\left\{\begin{array}{lll}
j_{1} & j_{2} & J \\
l_{2} & l_{1} & s
\end{array}\right\} .
\end{aligned}
$$

This relation is incorrect in deShalit and Talmi (p. 520). 
We can combine the above expression with $s=\frac{1}{2}$ and Eq. A.7 to evaluate a frequentlyoccurring combination of $3-\mathrm{j}$ and $9-\mathrm{j}$ symbols:

$$
\begin{aligned}
& \sqrt{\left(2 l_{1}+1\right)\left(2 l_{2}+1\right)}\left(\begin{array}{ccc}
l_{2} & l_{1} & J \\
0 & 0 & 0
\end{array}\right)\left\{\begin{array}{ccc}
\frac{1}{2} & \frac{1}{2} & 1 \\
l_{1} & l_{2} & J \\
j_{1} & j_{2} & J
\end{array}\right\}=\frac{1}{\sqrt{6}}(-1)^{l_{1}-j_{1}+\frac{1}{2}} \\
& \times \frac{l_{1}\left(l_{1}+1\right)-l_{2}\left(l_{2}+1\right)-j_{1}\left(j_{1}+1\right)+j_{2}\left(j_{2}+1\right)}{\sqrt{J(J+1)(2 J+1)}}\left(\begin{array}{ccc}
j_{2} & J & j_{1} \\
-\frac{1}{2} & 0 & \frac{1}{2}
\end{array}\right) .
\end{aligned}
$$

\section{A.4 Rotation Operators and Matrices}

All of the usual discussion of rotations follows from the observation that the operator

$$
e^{-i \theta \mathbf{J} \cdot \hat{\mathbf{n}}}
$$

when applied to a state vector representing an object, results in an active rotation of the object by the angle $\theta$ about the direction $\hat{\mathbf{n}}$. By an active rotation, we mean that the same coordinate system is used before and after the rotation, and that the object moves to a new position within that frame. This and all rotations indicated below take place in a righthanded sense. We find active rotations to be most convenient, but consider both active and passive rotations below.

We now describe an active rotation of an object through the Euler angles $(\alpha, \beta, \gamma)$. To do this, we imagine a coordinate system fixed in the object, and this coordinate system, together with the object, is rotated three times in the following sequence. The axes of the body-fixed coordinate system originally point in the directions $\left(\hat{\mathbf{x}}_{\mathbf{0}}, \hat{\mathbf{y}}_{\mathbf{0}}, \hat{\mathbf{z}}_{\mathbf{0}}\right)$.

- We rotate by $\alpha$ about the original $z$ axis; the axes of the moving coordinate system then point in directions $\left(\hat{\mathbf{x}}_{\mathbf{1}}, \hat{\mathbf{y}}_{\mathbf{1}}, \hat{\mathbf{z}}_{\mathbf{1}}\right)$.

- We next rotate by $\beta$ about the new $y$ axis, $\hat{\mathbf{y}}_{\mathbf{1}}$; the axes of the moving coordinate system then point in directions $\left(\hat{\mathbf{x}}_{\mathbf{2}}, \hat{\mathbf{y}}_{\mathbf{2}}, \hat{\mathbf{z}}_{\mathbf{2}}\right)$.

- Finally we rotate by $\gamma$ about the new $z$ axis, $\hat{\mathbf{z}}_{2}$; the axes of the moving coordinate system then point in directions $\left(\hat{\mathbf{x}}_{\mathbf{3}}, \hat{\mathbf{y}}_{\mathbf{3}}, \hat{\mathbf{z}}_{\mathbf{3}}\right)$.

Assume that before rotation the object is represented by the function $A(\mathbf{r})$, where $\mathbf{r}$ indicates coordinates $(x, y, z)$ or $(r, \theta, \phi)$ relative to the original (space-fixed) system. Then after rotation the object will be represented by a new function of the same coordinates,

$$
A^{\prime}(\mathbf{r})=e^{-i J_{z 2} \gamma} e^{-i J_{y 1} \beta} e^{-i J_{z 0} \alpha} A(\mathbf{r})
$$

where $J_{z 2} \equiv \mathbf{J} \cdot \hat{\mathbf{z}}_{\mathbf{2}}$, etc. Since the same rotation may be accomplished by performing the rotations is the reversed order about the axes in the original (space-fixed) system, the last expression is equivalent to

$$
A^{\prime}(\mathbf{r})=e^{-i J_{z 0} \alpha} e^{-i J_{y 0} \beta} e^{-i J_{z 0} \gamma} A(\mathbf{r}) .
$$


The last expression is the important one for active rotations. We now derive an expression for a passive rotation. In this case, the same rotations are involved, but the object remains fixed in its original position, and we look for its description in the new rotated coordinate system. We start with the active rotation described above, and note that by construction the object in its new, rotated, position is represented by the original function of the new coordinates:

$$
A\left(\mathbf{r}^{\prime}\right)=A^{\prime}(\mathbf{r})
$$

We now make an active rotation of the object back to its original position by carrying out the three rotations in the opposite order:

$$
A(\mathbf{r})=e^{-i J_{z 0}(-\alpha)} e^{-i J_{y 1}(-\beta)} e^{-i J_{z 2}(-\gamma)} A\left(\mathbf{r}^{\prime}\right) .
$$

Noting that in each of the three rotations the axis of rotation remains unchanged, we can also write this as

$$
A(\mathbf{r})=e^{-i J_{z 1}(-\alpha)} e^{-i J_{y 2}(-\beta)} e^{-i J_{z 3}(-\gamma)} A\left(\mathbf{r}^{\prime}\right),
$$

which is equivalent to carrying out the rotations in the reverse order about the axes in the $\left(\hat{\mathbf{x}}_{\mathbf{3}}, \hat{\mathbf{y}}_{\mathbf{3}}, \hat{\mathbf{z}}_{\mathbf{3}}\right)$ system,

$$
A(\mathbf{r})=e^{i J_{z 3} \gamma} e^{i J_{y 3} \beta} e^{i J_{z 3} \alpha} A\left(\mathbf{r}^{\prime}\right) .
$$

This is the important expression describing a passive rotation. Note that the same function appears on both sides of the equation, but that the coordinates refer to the original system on the left and the rotated system on the right. The rotations are made about axes defined in the rotated system.

We use the definition of Edmonds [2] for the rotation operator,

$$
R(\alpha, \beta, \gamma)=e^{i J_{z} \alpha} e^{i J_{y} \beta} e^{i J_{z} \gamma}
$$

This allows us to write the active rotation as

$$
A\left(\mathbf{r}^{\prime}\right)=A^{\prime}(\mathbf{r})=R^{(0)}(-\alpha,-\beta,-\gamma) A(\mathbf{r})
$$

and the passive rotation as

$$
A(\mathbf{r})=R^{(3)}(\gamma, \beta, \alpha) A\left(\mathbf{r}^{\prime}\right)
$$

where the superscripts on $R$ indicate that the rotations are defined with respect to the original coordinate system for the active rotation, and with respect to the rotated system for the passive rotation.

We use Edmonds' definition for the rotation matrices describing the transformations among states with angular momentum eigenvalue $j$,

$$
\mathcal{D}_{m m^{\prime}}^{j}(\alpha, \beta, \gamma)=\left\langle j m|R(\alpha, \beta, \gamma)| j m^{\prime}\right\rangle,
$$

where all components within the matrix element must be described in the same coordinate system. Then the active transformation of a state $|j m(0)\rangle$ yields the state

$$
|j m(3)\rangle=\sum_{m^{\prime}}\left|j m^{\prime}(0)\right\rangle \mathcal{D}_{m^{\prime} m}^{j}(-\alpha,-\beta,-\gamma),
$$


where $|j m(3)\rangle$ is the state after it has been actively rotated so that its projection on the new (body-fixed) $z$ axis is the same as it was on the original (space-fixed) $z$ axis. The right hand side gives its expansion in the basis defined in the space-fixed system. The passive rotation expresses the wave function with projection $m$ on the original $z$ axis in terms of an expansion in the basis functions in the rotated system:

$$
|j m(0)\rangle=\sum_{m^{\prime}}\left|j m^{\prime}(3)\right\rangle \mathcal{D}_{m^{\prime} m}^{j}(\gamma, \beta, \alpha)
$$

Definitions of the rotation operator and $\mathcal{D}$ function vary among authors. We show here the $\mathcal{D}$ functions as defined by

- Ed: Edmonds [2]

- BM: Bohr and Mottelson [13]

- BS: Brink and Satchler [14]

- Ro: Rose [15]

These definitions are

$$
\begin{aligned}
\mathcal{D}_{m m^{\prime}}^{j}(\alpha, \beta, \gamma)_{E d} & =\left\langle j m\left|e^{i J_{z} \alpha} e^{i J_{y} \beta} e^{i J_{z} \gamma}\right| j m^{\prime}\right\rangle \\
\mathcal{D}_{m m^{\prime}}^{j}(\alpha, \beta, \gamma)_{B M} & =\left\langle j m\left|e^{-i J_{z} \alpha} e^{-i J_{y} \beta} e^{-i J_{z} \gamma}\right| j m^{\prime}\right\rangle^{*} \\
\mathcal{D}_{m m^{\prime}}^{j}(\alpha, \beta, \gamma)_{B S} & =\left\langle j m\left|e^{-i J_{z} \alpha} e^{-i J_{y} \beta} e^{-i J_{z} \gamma}\right| j m^{\prime}\right\rangle \\
\mathcal{D}_{m m^{\prime}}^{j}(\alpha, \beta, \gamma)_{R o} & =\left\langle j m\left|e^{-i J_{z} \alpha} e^{-i J_{y} \beta} e^{-i J_{z} \gamma}\right| j m^{\prime}\right\rangle .
\end{aligned}
$$

Note that the definitions of Brink and Satchler and of Rose are identical. To convert to the Edmonds notation the following relations are useful:

$$
\begin{aligned}
\mathcal{D}_{m m^{\prime}}^{j}(\alpha, \beta, \gamma)_{B M} & =\mathcal{D}_{m m^{\prime}}^{j *}(-\alpha,-\beta,-\gamma)_{E d}=(-1)^{m^{\prime}-m} \mathcal{D}_{m m^{\prime}}^{j}(\alpha, \beta, \gamma)_{E d} \\
\mathcal{D}_{m m^{\prime}}^{j}(\alpha, \beta, \gamma)_{B S} & =\mathcal{D}_{m m^{\prime}}^{j}(-\alpha,-\beta,-\gamma)_{E d}=\mathcal{D}_{m^{\prime} m}^{j *}(\gamma, \beta, \alpha)_{E d} \\
\mathcal{D}_{m m^{\prime}}^{j}(\alpha, \beta, \gamma)_{R o} & =\mathcal{D}_{m m^{\prime}}^{j}(-\alpha,-\beta,-\gamma)_{E d}=\mathcal{D}_{m^{\prime} m}^{j *}(\gamma, \beta, \alpha)_{E d}
\end{aligned}
$$

\section{A.5 Reduced Matrix Elements}

We show here the relations between some commonly found definitions for the reduced matrix elements. Abbreviations and sources of these are

- Ed: Edmonds [2]

- BM: Bohr and Mottelson [13]

- BS: Brink and Satchler [14]

- Ro: Rose [15] 
The definitions of the reduced matrix element from the above sources can be written as

$$
\begin{aligned}
\left\langle j^{\prime} m^{\prime}\left|T_{k q}\right| j m\right\rangle & =(-1)^{2 k} \frac{1}{\sqrt{2 j^{\prime}+1}}\left(j m k q \mid j^{\prime} m^{\prime}\right)\left(j^{\prime}|| T_{k} \| j\right)_{E d} \\
& =(-1)^{2 k}\left(j m k q \mid j^{\prime} m^{\prime}\right)\left(j^{\prime}|| T_{k}|| j\right)_{B S} \\
& =\frac{1}{\sqrt{2 j^{\prime}+1}}\left(j m k q \mid j^{\prime} m^{\prime}\right)\left(j^{\prime}|| T_{k} \| j\right)_{B M} \\
& =\left(j m k q \mid j^{\prime} m^{\prime}\right)\left(j^{\prime}|| T_{k} \| j\right)_{R o} .
\end{aligned}
$$

To convert the other forms to the Edmonds form, which is normally used in these notes, we have

$$
\begin{aligned}
\left(j^{\prime}|| T_{k} \| j\right)_{B S} & =\frac{1}{\sqrt{2 j^{\prime}+1}}\left(j^{\prime}\left\|T_{k}\right\| j\right)_{E d} \\
\left(j^{\prime}\left\|T_{k}\right\| j\right)_{B M} & =(-1)^{2 k}\left(j^{\prime}\left\|T_{k}\right\| j\right)_{E d} \\
\left(j^{\prime}\left\|T_{k}\right\| j\right)_{R o} & =(-1)^{2 k} \frac{1}{\sqrt{2 j^{\prime}+1}}\left(j^{\prime}\left\|T_{k}\right\| j\right)_{E d}
\end{aligned}
$$

\section{A.6 B Expressions for Transition Strengths}

From Bohr and Mottelson Vol. 1, p. 37 [13] we find the definition of $B$ and its expression in terms of the reduced matrix element. We show this definition and its expression in terms of several definitions of the reduced matrix element as indicated in Sec. A.5:

$$
\begin{aligned}
B\left(T_{\lambda} ; I_{1} \rightarrow I_{2}\right) & =\sum_{\mu M_{2}}\left|\left\langle I_{2} M_{2}\left|T_{\lambda \mu}\right| I_{1} M_{1}\right\rangle\right|^{2} \\
& =\frac{1}{2 I_{1}+1}\left|\left(I_{2}|| T_{\lambda}|| I_{1}\right)_{B M, E d}\right|^{2} \\
& =\frac{2 I_{2}+1}{2 I_{1}+1}\left|\left(I_{2}|| T_{\lambda}|| I_{1}\right)_{B S}\right|^{2}
\end{aligned}
$$

Although the operator $T_{\lambda}$ may be any spherical tensor, a common choice is the multipole operator, which can be expressed as a sum over protons and neutrons as

$$
T_{\lambda \mu}=c_{p} \sum_{p} r_{p}^{\lambda} Y_{\lambda \mu}\left(\hat{\mathbf{r}}_{p}\right)+c_{n} \sum_{n} r_{n}^{\lambda} Y_{\lambda \mu}\left(\hat{\mathbf{r}}_{n}\right),
$$

where the constants $c_{p}, c_{n}$ may be chosen to yield the charge, isoscalar (or mass), isovector, proton, or neutron multipole operators. It is important to remember to include kinematic effective charges in these constants if appropriate. 


\section{Appendix B}

\section{Spectroscopy in Second Quantization}

\section{B.1 Second Quantization and $Z$ Amplitudes}

An arbitrary one-body spherical tensor operator acting in a many-body nuclear system can be represented in second quantization as

$$
\begin{aligned}
& O_{J M} \mathcal{T}_{T q}=\sum_{\substack{\alpha_{1} j_{1} m_{1} t_{1} \\
\alpha_{2} j_{2} m_{2} t_{2}}}\left\langle\alpha_{2} j_{2} m_{2} t_{2}\left|O_{J M} \mathcal{T}_{T q}\right| \alpha_{1} j_{1} m_{1} t_{1}\right\rangle a_{\alpha_{2} j_{2} m_{2} t_{2}}^{\dagger} a_{\alpha_{1} j_{1} m_{1} t_{1}} \\
&=\sum_{\substack{\alpha_{1} j_{1} m_{1} t_{1} \\
\alpha_{2} j_{2} m_{2} t_{2}}} \frac{1}{\sqrt{2 J+1}}(-1)^{j_{1}-m_{1}}\left(j_{2} m_{2} j_{1}-m_{1} \mid J M\right) \\
& \times\left(\alpha_{2} j_{2} t_{2}|| O_{J}|| \alpha_{1} j_{1} t_{1}\right) a_{\alpha_{2} j_{2} m_{2} t_{2}}^{\dagger} a_{\alpha_{1} j_{1} m_{1} t_{1}} \\
& \times\left\langle\frac{1}{2} t_{2}\left|\mathcal{T}_{T q}\right| \frac{1}{2} t_{1}\right\rangle .
\end{aligned}
$$

$O_{J M}$ is a space-spin operator and $\mathcal{T}_{T q}$ is an isospin operator. In representing the singleparticle basis we have explicitly specified the isospin projection $t\left(\frac{1}{2}\right.$ for neutrons and $-\frac{1}{2}$ for protons), as well as the total angular momentum and projection $j m$. All other necessary quantum numbers are abbreviated by $\alpha$. Typically this includes $n$, the principal quantum number, and $l$, the orbital angular momentum, with the spin of value $\frac{1}{2}$ understood. The isospin operator is defined so that $\mathcal{T}_{00}=1$, and the quantities $\mathcal{T}_{1 q}$ are the spherical components of the vector of Pauli matrices $\boldsymbol{\tau}$. Note that in the second line above, the matrix element has been reduced in angular momentum but not in isospin. We retain the isospin labels $t_{1}, t_{2}$ in the reduced matrix element because the radial wave functions may depend on whether the particle is a neutron or proton.

For further use, we define an abbreviation for the isospin matrix element,

$$
\mathcal{T}_{T q}^{t_{2} t_{1}}=\left\langle\frac{1}{2} t_{2}\left|\mathcal{T}_{T q}\right| \frac{1}{2} t_{1}\right\rangle
$$

Values of the isospin matrix element $\mathcal{T}_{T q}^{t_{2} t_{1}}$ are shown in Table B.1.

We define a spherical tensor operator in Fock space by

$$
A_{t_{2} t_{1}}^{J M}\left(\alpha_{2} j_{2}, \alpha_{1} j_{1}\right)=\sum_{m_{1} m_{2}}(-1)^{j_{1}-m_{1}}\left(j_{2} m_{2} j_{1}-m_{1} \mid J M\right) a_{\alpha_{2} j_{2} m_{2} t_{2}}^{\dagger} a_{\alpha_{1} j_{1} m_{1} t_{1}} .
$$




\begin{tabular}{|rrrcr|}
\hline$t_{2}$ & $t_{1}$ & $q$ & $\mathcal{T}_{0 q}^{t_{2} t_{1}}$ & $\mathcal{T}_{1 q}^{t_{2} t_{1}}$ \\
\hline$\frac{1}{2}$ & $\frac{1}{2}$ & 0 & 1 & 1 \\
$\frac{1}{2}$ & $-\frac{1}{2}$ & 1 & 0 & $-\sqrt{2}$ \\
$-\frac{1}{2}$ & $\frac{1}{2}$ & -1 & 0 & $\sqrt{2}$ \\
$-\frac{1}{2}$ & $-\frac{1}{2}$ & 0 & 1 & -1 \\
\hline
\end{tabular}

Table B.1: Values of the isospin matrix element of Eq. B.3. Note that $q=t_{2}-t_{1}$.

This operator is a spherical tensor because it is the vector coupling of two spherical tensors of rank and projection $j_{2} m_{2}$ and $j_{1} m_{1}$,

$$
a_{\alpha_{2} j_{2} m_{2} t_{2}}^{\dagger} \quad \text { and } \quad(-1)^{j_{1}+m_{1}} a_{\alpha_{1} j_{1}-m_{1} t_{1}}
$$

as can be seen by replacing $m_{1}$ by $-m_{1}$ everywhere in the defining equation for $A_{t_{2} t_{1}}^{J M}$. The one-body operator may now be written as

$$
O_{J M} \mathcal{T}_{T q}=\sum_{\substack{\alpha_{1} j_{1} t_{1} \\ \alpha_{2} j_{2} t_{2}}} \frac{1}{\sqrt{2 J+1}}\left(\alpha_{2} j_{2} t_{2}\left\|O_{J}\right\| \alpha_{1} j_{1} t_{1}\right) A_{t_{2} t_{1}}^{J M}\left(\alpha_{2} j_{2}, \alpha_{1} j_{1}\right) \mathcal{T}_{T q}^{t_{2} t_{1}}
$$

The spectroscopic amplitudes $Z_{t_{2} t_{1}}^{J}$ are defined in terms of the reduced matrix elements of the $A_{t_{2} t_{1}}^{J M}$ operators between nuclear states $\alpha_{i} I_{i}$ and $\alpha_{f} I_{f}$, where again $\alpha_{i, f}$ signify extra quantum numbers needed to specify the states completely, including any necesary isospin quantum numbers. The definition is:

$$
Z_{t_{2} t_{1}}^{J}\left(\alpha_{2} j_{2}, \alpha_{1} j_{1}\right)=\frac{1}{\sqrt{(2 J+1)\left(2 I_{i}+1\right)}}\left(\alpha_{f} I_{f}\left\|A_{t_{2} t_{1}}^{J}\left(\alpha_{2} j_{2}, \alpha_{1} j_{1}\right)\right\| \alpha_{i} I_{i}\right),
$$

where explicit reference to the initial and final states is not indicated on the left; this must be remembered. We can now express the reduced matrix elements of the one body operator between complete nuclear states in terms of reduced matrix elements between single-particle states and spectroscopic amplitudes:

$$
\left(\alpha_{f} I_{f}\left\|O_{J} \mathcal{T}_{T q}\right\| \alpha_{i} I_{i}\right)=\sum_{\substack{\alpha_{1} j_{1} t_{1} \\ \alpha_{2} j_{2} t_{2}}} \sqrt{2 I_{i}+1} Z_{t_{2} t_{1}}^{J}\left(\alpha_{2} j_{2}, \alpha_{1} j_{1}\right)\left(\alpha_{2} j_{2} t_{2}\left\|O_{J}\right\| \alpha_{1} j_{1} t_{1}\right) \mathcal{T}_{T q}^{t_{2} t_{1}}
$$

The above definitions for the $A$ operators and $Z$ coefficients are discussed by Petrovich et al. in the appendix to Ref. [16], along with comparisons of the notations and normalizations of equivalent quantities used by other authors. In particular, we have chosen to work in the so-called "p-n" scheme in which the individual nucleons are not coupled to total isospin. Ref. [16] also discusses the case in which they are coupled to total isospin and the reduced matrix elements are reduced in both angular momentum and isospin.

It is sometimes convenient to make a canonical transformation to particle and hole creation and destruction operators in which, for orbitals below the Fermi energy, we define

$$
b_{\alpha j m t}^{\dagger}=(-1)^{j \pm m} a_{\alpha j-m t} .
$$


The sign in the phase factor varies among authors; we choose the upper sign. $b_{\alpha j m t}^{\dagger}$ is a spherical tensor of rank and projection $j m$.

The definition of the $Z$ amplitudes is chosen so that for excitation of a single particle-hole pair (without charge exchange) coupled to spin $J$ starting from a closed spin- 0 core, the $Z$ amplitude is unity, within a phase that can be chosen as \pm 1 . That is, for use in Eq. B.7, we choose

$$
\left|\alpha_{i} I_{i} M_{i}\right\rangle \rightarrow|00\rangle \quad \text { and } \quad\left|\alpha_{f} I_{f} M_{f}\right\rangle \rightarrow \pm A_{t t}^{I_{f} M_{f}}\left(\alpha_{2}^{\prime} j_{2}^{\prime}, \alpha_{1}^{\prime} j_{1}^{\prime}\right)|00\rangle
$$

Working this out by inserting B.4 and B.10 into B.7 leads to

$$
Z_{t_{2} t_{1}}^{J}\left(\alpha_{2} j_{2}, \alpha_{1} j_{1}\right)= \pm \delta_{J I_{f}} \delta_{\alpha_{2} j_{2} t_{2}, \alpha_{2}^{\prime} j_{2}^{\prime} t} \delta_{\alpha_{1} j_{1} t_{1}, \alpha_{1}^{\prime} j_{1}^{\prime} t}
$$

The choice of sign in the preceding two equations corresponds to the choice of sign in the defining relation for the hole operators in Eq. B.9.

We can also find a simple expression for the $Z$ amplitude for a transition between two orbitals outside a closed core (again without charge exchange). In this case we choose

$$
\left|\alpha_{i} I_{i} M_{i}\right\rangle \rightarrow a_{\alpha_{i} j_{i} m_{i} t}^{\dagger}|00\rangle \quad \text { and } \quad\left|\alpha_{f} I_{f} M_{f}\right\rangle \rightarrow a_{\alpha_{f} j_{f} m_{f} t}^{\dagger}|00\rangle
$$

and the result is

$$
Z_{t_{2} t_{1}}^{J}\left(\alpha_{2} j_{2}, \alpha_{1} j_{1}\right)=\frac{1}{\sqrt{2 j_{i}+1}} \delta_{\alpha_{2} j_{2} t_{2}, \alpha_{f} j_{f} t} \delta_{\alpha_{1} j_{1} t_{1}, \alpha_{i} j_{i}}
$$

where $J$ can range from $\left|j_{i}-j_{f}\right|$ to $j_{i}+j_{f}$.

Let us consider the effect of a change in the single-particle basis. Since we consider the total angular momentum, its $z$-axis projection, and the isospin projection to be conserved, we can consider the transformation to connect one set of quantum numbers $\alpha$ with another set $\beta$. Thus

$$
|\alpha j m t\rangle=\sum_{\beta}|\beta j m t\rangle\langle\beta j m t \mid \alpha j m t\rangle \equiv \sum_{\beta}|\beta j m t\rangle\langle\beta \mid \alpha\rangle_{j t},
$$

where in the definition on the right we recognize that the transformation coefficients are independent of $m$. As an example, the $\alpha$ states might represent a basis of Hartree-Fock states and the $\beta$ states spherical harmonic oscillator states. The treatment shown here can be easily generalized for other expansions in which $j$ is not conserved, such as in the expansion of deformed-potential single-particle states on a spherical basis. We can therefore rewrite Eq. B.8 as

$$
\begin{aligned}
& \left(\alpha_{f} I_{f}\left\|O_{J} \mathcal{T}_{T q}\right\| \alpha_{i} I_{i}\right)=\sum_{\substack{\alpha_{1} \beta_{1} j_{1} t_{1} \\
\alpha_{2} \beta_{2} j_{2} t_{2}}} \sqrt{2 I_{i}+1} Z_{t_{2} t_{1}}^{J}\left(\alpha_{2} j_{2}, \alpha_{1} j_{1}\right) \\
& \quad \times\left\langle\alpha_{2} \mid \beta_{2}\right\rangle_{j_{2} t_{2}}\left(\beta_{2} j_{2} t_{2}|| O_{J}|| \beta_{1} j_{1} t_{1}\right)\left\langle\beta_{1} \mid \alpha_{1}\right\rangle_{j_{1} t_{1}} \mathcal{T}_{T q}^{t_{2} t_{1}} .
\end{aligned}
$$

We see from this last equation that we can redefine the $Z$ amplitudes in the new basis,

$$
\left(\alpha_{f} I_{f}\left\|O_{J} \mathcal{T}_{T q}\right\| \alpha_{i} I_{i}\right)=\sum_{\substack{\beta_{1} j_{1} t_{1} \\ \beta_{2} j_{2} t_{2}}} \sqrt{2 I_{i}+1} Z_{t_{2} t_{1}}^{J}\left(\beta_{2} j_{2}, \beta_{1} j_{1}\right)\left(\beta_{2} j_{2} t_{2}\left\|O_{J}\right\| \beta_{1} j_{1} t_{1}\right) \mathcal{T}_{T q}^{t_{2} t_{1}}
$$

where

$$
Z_{t_{2} t_{1}}^{J}\left(\beta_{2} j_{2}, \beta_{1} j_{1}\right)=\sum_{\alpha_{1} \alpha_{2}}\left\langle\alpha_{2} \mid \beta_{2}\right\rangle_{j_{2} t_{2}} Z_{t_{2} t_{1}}^{J}\left(\alpha_{2} j_{2}, \alpha_{1} j_{1}\right)\left\langle\beta_{1} \mid \alpha_{1}\right\rangle_{j_{1} t_{1}}
$$




\section{B.2 Evaluation of Single-Particle Matrix Elements}

In this section we work out the expressions for the single-particle matrix elements of an important class of operators, which have the form

$$
O_{L S J M} \mathcal{T}_{T q}=f_{L S J}(r)\left[Y_{L}(\hat{\mathbf{r}}) \times \mathcal{S}_{S}\right]_{J M} \mathcal{T}_{T q}
$$

where $S=0$ or 1 . The operators $\mathcal{S}_{S \nu}$ are defined by

$$
\mathcal{S}_{00}=1 \quad \text { and } \quad \mathcal{S}_{1 \nu}=\sigma_{1 \nu}
$$

where the quantities $\sigma_{1 \nu}$ are the spherical components of the Pauli operator $\boldsymbol{\sigma}$. Similarly, the isospin operators $\mathcal{T}_{T q}$ are defined by

$$
\mathcal{T}_{00}=1 \quad \text { and } \quad \mathcal{T}_{1 q}=\tau_{1 q}
$$

where the quantities $\tau_{1 q}$ are the spherical components of the Pauli operator $\boldsymbol{\tau}$.

The state vectors in a spherical basis are described by

$$
|n l s j m t\rangle=\frac{u_{n l j t}(r)}{r} i^{l}\left[Y_{l}(\hat{\mathbf{r}}) \times \chi_{\frac{1}{2}}\right]_{j m} \chi_{\frac{1}{2} t}^{i s o} .
$$

Note, in particular, the inclusion of the $i^{l}$ factor and the choice of coupling order. The index $s$ in the ket on the left always has the value $\frac{1}{2}$. The index $t$ on the isospinor $\chi_{\frac{1}{2} t}^{\text {iso }}$ is $\frac{1}{2}$ for neutrons and $-\frac{1}{2}$ for protons.

We evaluate the following matrix element, which is reduced in angular momentum but not in isospin:

$$
\begin{aligned}
\left(n_{2} l_{2} s_{2} j_{2} t_{2}\left\|f_{L S J}(r)\left[Y_{L}(\hat{\mathbf{r}}) \times \mathcal{S}_{S}\right]_{J} \mathcal{T}_{T q}\right\| n_{1} l_{1} s_{1} j_{1} t_{1}\right) & \\
=i^{l_{1}-l_{2}} & \int_{0}^{\infty} d r u_{n_{2} l_{2} j_{2} t_{2}}^{*}(r) f_{L S J}(r) u_{n_{1} l_{1} j_{1} t_{1}}(r) \\
& \times\left(l_{2} \frac{1}{2} j_{2}\left\|\left[Y_{L} \times \mathcal{S}_{S}\right]_{J}\right\| l_{1} \frac{1}{2} j_{1}\right) \mathcal{T}_{T q}^{t_{2} t_{1}}
\end{aligned}
$$

where the isospin matrix element is $\mathcal{T}_{T q}^{t_{2} t_{1}}=\left\langle\frac{1}{2} t_{2}\left|\mathcal{T}_{T q}\right| \frac{1}{2} t_{1}\right\rangle$; numerical values of this matrix element are shown in Table B.1. Eq. B.22 can be substituted for the combination of reduced matrix element and isospin matrix element on the right-hand side of Eq. B.8.

The reduced matrix element on the right can be factored using Edmonds, Eq. 7.1.5, p. 110 as

$$
\left(l_{2} \frac{1}{2} j_{2}\left\|\left[Y_{L} \times \mathcal{S}_{S}\right]_{J}\right\| l_{1} \frac{1}{2} j_{1}\right)=\hat{j_{1}} \hat{j_{2}} \hat{J}\left\{\begin{array}{ccc}
l_{2} & l_{1} & L \\
\frac{1}{2} & \frac{1}{2} & S \\
j_{2} & j_{1} & J
\end{array}\right\}\left(l_{2}\left\|Y_{L}\right\| l_{1}\right)\left(\frac{1}{2}\left\|\mathcal{S}_{S}\right\| \frac{1}{2}\right)
$$

and the two reduced matrix elements in this expression are evaluated using Edmonds, Eqs. 5.4 .4 and 5.4.6, p. 76 as

$$
\left(l_{2}|| Y_{L} \| l_{1}\right)=(-1)^{l_{2}} \frac{1}{\sqrt{4 \pi}} \hat{l_{1}} \hat{l_{2}} \hat{L}\left(\begin{array}{ccc}
l_{2} & L & l_{1} \\
0 & 0 & 0
\end{array}\right) .
$$


and

$$
\left(\frac{1}{2}\left\|S_{S}\right\| \frac{1}{2}\right)=\sqrt{2} \hat{S}
$$

The result is

$$
\left(l_{2} \frac{1}{2} j_{2}\left\|\left[Y_{L} \times \mathcal{S}_{S}\right]_{J}\right\| l_{1} \frac{1}{2} j_{1}\right)=\frac{1}{\sqrt{2 \pi}}(-1)^{l_{2}} \hat{l_{1}} \hat{l_{2}} \hat{j_{1}} \hat{j_{2}} \hat{L} \hat{S} \hat{J}\left(\begin{array}{ccc}
l_{2} & l_{1} & L \\
0 & 0 & 0
\end{array}\right)\left\{\begin{array}{ccc}
l_{2} & l_{1} & L \\
\frac{1}{2} & \frac{1}{2} & S \\
j_{2} & j_{1} & J
\end{array}\right\} .
$$

The following symmetry relation results from interchanging initial- and final-state quantum numbers:

$$
\left(l_{1} \frac{1}{2} j_{1} \|\left[Y_{L} \times \mathcal{S}_{S}\right]_{J}|| l_{2} \frac{1}{2} j_{2}\right)=(-1)^{L+S+J+j_{2}-j_{1}}\left(l_{2} \frac{1}{2} j_{2}\left\|\left[Y_{L} \times \mathcal{S}_{S}\right]_{J}\right\| l_{1} \frac{1}{2} j_{1}\right) .
$$

We now write the expressions for the two possible values of $S$. For $S=0$, the 9 -j symbol in Eq. B.23 reduces to a 6 -j, and we require $L=J$. Then Eq. B.23 simplifies to

$$
\begin{aligned}
\left(l_{2} \frac{1}{2} j_{2}\left\|Y_{J}\right\| l_{1} \frac{1}{2} j_{1}\right) & =\frac{1}{\sqrt{4 \pi}}(-1)^{j_{1}+\frac{1}{2}+J} \hat{l_{1}} \hat{l_{2}} \hat{j_{1}} \hat{j_{2}} \hat{J}\left(\begin{array}{ccc}
l_{2} & l_{1} & J \\
0 & 0 & 0
\end{array}\right)\left\{\begin{array}{ccc}
l_{2} & j_{2} & \frac{1}{2} \\
j_{1} & l_{1} & J
\end{array}\right\} \\
& =\frac{1}{\sqrt{4 \pi}}(-1)^{\frac{1}{2}-j_{2}+J} \bar{Z}\left(l_{2} j_{2} l_{1} j_{1} ; \frac{1}{2} J\right),
\end{aligned}
$$

where the $\bar{Z}$ coefficient is defined in Eq. A.5. A further simplification is found by using a special relation involving 3-j and 6-j symbols (see Eq. A.7), which leads to:

$$
\left(l_{2} \frac{1}{2} j_{2} \| Y_{J}|| l_{1} \frac{1}{2} j_{1}\right)=\frac{1}{\sqrt{4 \pi}}(-1)^{j_{1}-\frac{1}{2}+J} \hat{j_{1}} \hat{j_{2}} \hat{J}\left(\begin{array}{ccc}
j_{2} & J & j_{1} \\
-\frac{1}{2} & 0 & \frac{1}{2}
\end{array}\right) .
$$

For $S=1$ the general result is

$$
\left(l_{2} \frac{1}{2} j_{2}\left\|\left[Y_{L} \times \boldsymbol{\sigma}\right]_{J}\right\| l_{1} \frac{1}{2} j_{1}\right)=\sqrt{\frac{3}{2 \pi}}(-1)^{l_{2}} \hat{l_{1}} \hat{l_{2}} \hat{j_{1}} \hat{j_{2}} \hat{L} \hat{J}\left(\begin{array}{ccc}
l_{2} & l_{1} & L \\
0 & 0 & 0
\end{array}\right)\left\{\begin{array}{ccc}
l_{2} & l_{1} & L \\
\frac{1}{2} & \frac{1}{2} & 1 \\
j_{2} & j_{1} & J
\end{array}\right\} .
$$

For $L \neq J$ no further simplification is possible, but if $L=J$ we can use a special relation for the combination of $3-\mathrm{j}$ and $9-\mathrm{j}$ symbols (Eq. A.10) to yield

$$
\left(l_{2} \frac{1}{2} j_{2}\left\|\left[Y_{J} \times \boldsymbol{\sigma}\right]_{J}\right\| l_{1} \frac{1}{2} j_{1}\right)=\frac{1}{\sqrt{4 \pi}} \xi\left(l_{1} l_{2} j_{1} j_{2} J\right)(-1)^{j_{1}-\frac{1}{2}+J} \hat{j_{1}} \hat{j_{2}} \hat{J}\left(\begin{array}{ccc}
j_{2} & J & j_{1} \\
-\frac{1}{2} & 0 & \frac{1}{2}
\end{array}\right),
$$

where we have defined

$$
\xi\left(l_{1} l_{2} j_{1} j_{2} J\right)=\frac{l_{1}\left(l_{1}+1\right)-l_{2}\left(l_{2}+1\right)-j_{1}\left(j_{1}+1\right)+j_{2}\left(j_{2}+1\right)}{\sqrt{J(J+1)}} .
$$

Note that $\left(l_{2} \frac{1}{2} j_{2}\left\|\left[Y_{J} \times \boldsymbol{\sigma}\right]_{J}\right\| l_{1} \frac{1}{2} j_{1}\right)=\xi\left(l_{1} l_{2} j_{1} j_{2} J\right)\left(l_{2} \frac{1}{2} j_{2}\left\|Y_{J}\right\| l_{1} \frac{1}{2} j_{1}\right)$.

If the coupling order of the orbital and spin angular momenta in the single-particle states is reversed, it is easy to see that

$$
\left(\frac{1}{2} l_{2} j_{2}\left\|\left[Y_{L} \times \mathcal{S}_{S}\right]_{J}\right\| \frac{1}{2} l_{1} j_{1}\right)=(-1)^{j_{2}-j_{1}+l_{2}-l_{1}}\left(l_{2} \frac{1}{2} j_{2}\left\|\left[Y_{L} \times \mathcal{S}_{S}\right]_{J}\right\| l_{1} \frac{1}{2} j_{1}\right) .
$$

Also, if the coupling order in the operator is reversed, we find

$$
\left(l_{2} \frac{1}{2} j_{2} \|\left[\mathcal{S}_{S} \times Y_{L}\right]_{J}|| l_{1} \frac{1}{2} j_{1}\right)=(-1)^{L+S-J}\left(l_{2} \frac{1}{2} j_{2}\left\|\left[Y_{L} \times \mathcal{S}_{S}\right]_{J}\right\| l_{1} \frac{1}{2} j_{1}\right) .
$$




\section{Appendix C}

\section{Fourier-Bessel Expansions}

\section{C.1 Introductory Remarks}

The following discussion is based on the treatment of Petrovich [17], which has been applied to inelastic scattering by Petrovich, Carr, and McManus in Ref. [1].

\section{C.2 Transformation of Partial-Wave Functions}

We consider the Fourier-Bessel series expansion of a function that can be expressed in the form

$$
f(\mathbf{r})=f_{J M}(r) Y_{J M}^{*}(\hat{\mathbf{r}}),
$$

which is defined within a radius $R$. We refer to the radial part $f_{J M}(r)$ as a partial-wave function. In applications the function $f_{J M}$ is frequently independent of $M$ and is then labeled $f_{J}$; we retain the more general form. The Fourier transform is defined as

$$
f(\mathbf{q})=\int d \mathbf{r} e^{ \pm i \mathbf{q} \cdot \mathbf{r}} f(\mathbf{r}),
$$

where the domain of the radial part of the integral is 0 to $R$. Petrovich employs the lower (-) sign; we retain both possibilities.

We use the expansion

$$
\begin{aligned}
e^{ \pm i \mathbf{q} \cdot \mathbf{r}} & =4 \pi \sum_{J M}( \pm i)^{J} j_{J}(q r) Y_{J M}(\hat{\mathbf{r}}) Y_{J M}^{*}(\hat{\mathbf{q}}) \\
& =4 \pi \sum_{J M}( \pm i)^{J} j_{J}(q r) Y_{J M}^{*}(\hat{\mathbf{r}}) Y_{J M}(\hat{\mathbf{q}}) \\
& =4 \pi \sum_{J}( \pm i)^{J} j_{J}(q r) Y_{J}^{*}(\hat{\mathbf{r}}) \cdot Y_{J}(\hat{\mathbf{q}}),
\end{aligned}
$$

where in the last line we have used the definition of the dot product of spherical tensors (see, for example, Edmonds [2]). Inserting this in Eq. C.2 we find

$$
f(\mathbf{q})=( \pm i)^{J} f_{J M}(q) Y_{J M}^{*}(\hat{\mathbf{q}}),
$$


where we have defined

$$
f_{J M}(q)=4 \pi \int_{0}^{R} d r r^{2} j_{J}(q r) f_{J M}(r),
$$

which is the partial-wave form of the transformation; we refer to the function $f_{J M}(q)$ as the partial-wave function in momentum space.

The reverse transformation is given by

$$
f(\mathbf{r})=\sum_{n=1}^{\infty} w_{n}^{2} \int d \hat{\mathbf{q}}_{n} e^{\mp i \mathbf{q}_{n} \cdot \mathbf{r}} f\left(\mathbf{q}_{n}\right),
$$

where $\left|\mathbf{q}_{n}\right| \equiv q_{n}=n \pi / R$, and $w_{n}^{2}=n^{2} /\left(8 R^{3}\right)=(2 \pi)^{-3} q_{n}^{2} \Delta q_{n}$, where $\Delta q_{n}=\pi / R$. By inserting Eqs. C.1, C.4, and C.6 into Eq. C.8 and carrying out the angular integral we find the partial-wave form of the reverse transformation,

$$
f_{J M}(r)=4 \pi \sum_{n=1}^{\infty} w_{n}^{2} j_{J}\left(q_{n} r\right) f_{J M}\left(q_{n}\right) .
$$

In the limit $R \rightarrow \infty$ it is easy to see that the expression for the reverse transformation is the usual Fourier integral; that is,

$$
f(\mathbf{r})=\frac{1}{(2 \pi)^{3}} \int d \mathbf{q} e^{\mp i \mathbf{q} \cdot \mathbf{r}} f(\mathbf{q}),
$$

where the integral extends over all momentum space, or in partial-wave form

$$
f_{J M}(r)=\frac{4 \pi}{(2 \pi)^{3}} \int_{0}^{\infty} d q q^{2} j_{J}(q r) f_{J M}(q) .
$$

Numerical calculations are more efficiently carried out using the series representation than the integral representation; examples are shown in Ref. [17]. The above expressions show how to convert from one form to the other. It is usually more convenient to carry out derivations of the expressions for physical applications in the integral representation rather than the series representation. In this case, we replace the upper limit $R$ on the radial integrals by $\infty$. When converting from the integral representation to the series representation, Eqs. C.9 and C.11 above show that it suffices to make the replacement

$$
\int_{0}^{\infty} d q q^{2}(\cdots) \rightarrow(2 \pi)^{3} \sum w_{n}^{2}(\cdots),
$$

together with a corresponding replacement of $q$ in the integrand by $q_{n}$ in the sum.

If the partial wave expansion of the function to be transformed is defined in terms of $Y_{J M}$ instead of $Y_{J M}^{*}$, the above discussion is unaltered. That is, if $Y_{J M}^{*}$ is replaced by $Y_{J M}$ in Eq. C.1, all expressions are identical except for the same replacement in Eq. C.6.

We can define alternative normalizations for the transformed function. For example, if we define the forward transformation as

$$
f_{J M}^{\prime}(q)=\int_{0}^{R} d r r^{2} j_{J}(q r) f_{J M}(r),
$$


so that $f_{J M}^{\prime}(q)=f_{J M}(q) /(4 \pi)$, then the reverse transformation may be written as

$$
f_{J M}(r)=\sum_{n=1}^{\infty} w_{n}^{\prime 2} j_{J}\left(q_{n} r\right) f_{J M}^{\prime}\left(q_{n}\right)
$$

where ${w^{\prime}}_{n}^{2}=(4 \pi)^{2} w_{n}^{2}=\left(2 \pi^{2} / R^{3}\right) n^{2}$. Thus this choice of normalization and definition of the weight factor gets rid of the explicit factors of $4 \pi$ preceding the integral or sum in the transformations.

\section{C.3 Transformation of Scalar Functions}

It is often useful to choose a different normalization for functions with $J M=00$; i.e. functions of the form

$$
v(\mathbf{r})=v(r),
$$

in which the factor $Y_{00}=1 / \sqrt{4 \pi}$ is omitted. We also omit this factor in the transformed function. Then $v(\mathbf{q})=v(q)$ may be expressed as

$$
v(q)=\int d \mathbf{r} e^{ \pm i \mathbf{q} \cdot \mathbf{r}} v(r)=4 \pi \int_{0}^{\infty} d r r^{2} j_{0}(q r) v(r),
$$

which is the same as the relation between the partial wave functions, Eq. C.7. We have here taken $R \rightarrow \infty$, since we assume that $v(r)$ is small beyond $R$, as will be the case for the nucleon-nucleon interaction in inelastic scattering calculations.

The transformed functions provide simple expressions for the volume integral $J$ and mean-square radius $\left\langle r^{2}\right\rangle$ of the function $v(r)$. The volume integral is obtained immediately by setting $q=0$ in Eq. C.16,

$$
J=v(q=0) .
$$

To get the m.s. radius, we expand the spherical Bessel function in Eq. C.16 as

$$
j_{0}(q r)=\frac{\sin (q r)}{q r}=1-\frac{1}{6} q^{2} r^{2}+\cdots .
$$

We insert this in Eq. C.16 and take the derivative of both sides with respect to $q^{2}$. This leads to the result

$$
\left\langle r^{2}\right\rangle=-\left.6 \frac{1}{J} \frac{d v(q)}{d\left(q^{2}\right)}\right|_{q=0} .
$$

The following is a collection of expressions for several frequently-encountered scalar functions, together with their volume integrals and mean square radii.

- Gaussian interaction:

$$
\begin{aligned}
v(r) & =-V_{0} e^{-\mu^{2} r^{2}} \\
v(q) & =-V_{0} \frac{1}{\mu^{3}} \pi^{3 / 2} e^{-q^{2} /(2 \mu)^{2}} \\
J & =-V_{0} \frac{1}{\mu^{3}} \pi^{3 / 2} \\
\left\langle r^{2}\right\rangle & =\frac{3}{2} \frac{1}{\mu^{2}}
\end{aligned}
$$


- Yukawa interaction:

$$
\begin{aligned}
v(r) & =-V_{0} e^{-\mu r} /(\mu r) \\
v(q) & =-V_{0} \frac{1}{\mu} \frac{4 \pi}{q^{2}+\mu^{2}} \\
J & =-V_{0} \frac{4 \pi}{\mu^{3}} \\
\left\langle r^{2}\right\rangle & =6 \frac{1}{\mu^{2}}
\end{aligned}
$$

- Zero-range interaction:

$$
\begin{aligned}
v(r) & =-J_{0} \delta(\mathbf{r}) \\
v(q) & =-J_{0} \\
J & =-J_{0} \\
\left\langle r^{2}\right\rangle & =0
\end{aligned}
$$

- Coulomb interaction ( $J$ and $\left\langle r^{2}\right\rangle$ undetermined):

$$
\begin{aligned}
& v(r)=Z_{1} Z_{2} e^{2} / r \\
& v(q)=4 \pi Z_{1} Z_{2} e^{2} / q^{2}
\end{aligned}
$$

- Woods-Saxon interaction, to be calculated numerically:

$$
\begin{aligned}
v(r) & =-V_{0} \frac{1}{1+\exp \left(\frac{r-R}{a}\right)} \\
v(q) & =-4 \pi V_{0} \int_{0}^{\infty} d r r^{2} \frac{1}{1+\exp \left(\frac{r-R}{a}\right)} j_{0}(q r) \\
J & =-4 \pi V_{0} \int_{0}^{\infty} d r r^{2} \frac{1}{1+\exp \left(\frac{r-R}{a}\right)} \\
\left\langle r^{2}\right\rangle & =-4 \pi V_{0} \frac{1}{J} \int_{0}^{\infty} d r r^{4} \frac{1}{1+\exp \left(\frac{r-R}{a}\right)}
\end{aligned}
$$

- Exponential function, frequently used to represent proton charge distribution normalized to unit volume integral:

$$
\begin{aligned}
v(r) & =\frac{\kappa^{3}}{8 \pi} e^{-\kappa r} \\
v(q) & =\left(1+\frac{q^{2}}{\kappa^{2}}\right)^{-2} \\
J & =1 \\
\left\langle r^{2}\right\rangle & =12 / \kappa^{2}
\end{aligned}
$$

The proton charge distribution is well described with $\kappa^{2}=18.774 \mathrm{fm}^{-2}$, which corresponds to $\left\langle r^{2}\right\rangle=0.63918 \mathrm{fm}^{2}$, or r.m.s. radius $0.79949 \mathrm{fm}$. 
It is useful to note the correspondence between the strength and inverse-range parameters of the Gaussian and Yukawa interactions that yields the same volume integral and meansquare radius:

$$
\begin{aligned}
V_{0}(\text { Yukawa }) & =\sqrt{4 \pi} V_{0}(\text { Gaussian }) \\
\mu(\text { Yukawa }) & =2 \mu(\text { Gaussian })
\end{aligned}
$$

\section{C.4 Convolution and Scaling Formulas}

Consider a function $U$ obtained by convolution of two functions $v$ and $f$; that is

$$
U(\mathbf{r})=\int d \mathbf{r}^{\prime} v\left(\mathbf{r}-\mathbf{r}^{\prime}\right) f\left(\mathbf{r}^{\prime}\right)
$$

If we transform this equation to momentum space by transforming each of the functions using Eqs. C.2,C.10, we obtain the well-known result

$$
U(\mathbf{q})=v(\mathbf{q}) f(\mathbf{q})
$$

We now specialize this general result to the case where $v$ is a scalar function as in Eq. C.15, and $f$ is a partial-wave function as given by Eq. C.1. Then $U$ is also a partial-wave function with the same values of $J, M$ as $f$, and the convolution function becomes

$$
U_{J M}(q)=v(q) f_{J M}(q)
$$

We also note a radial scaling law. If we define a function $F$ that is the Fourier transform of a function $f$ scaled by a parameter $\alpha$ by

$$
F_{\alpha}(\mathbf{q})=\int d \mathbf{r} f(\mathbf{r} / \alpha) e^{ \pm i \mathbf{q} \cdot \mathbf{r}}
$$

we easily find by changing the integration variable that

$$
F_{\alpha}(\mathbf{q})=\alpha F_{1}(\alpha \mathbf{q})
$$

This result applies also to the scalar and partial-wave forms, with obvious changes in notation.

\section{C.5 Delta Function}

The delta function can be expanded in spherical harmonics as

$$
\delta\left(\mathbf{r}-\mathbf{r}^{\prime}\right)=\frac{\delta\left(r-r^{\prime}\right)}{r r^{\prime}} \sum_{J M} Y_{J M}^{*}(\hat{\mathbf{r}}) Y_{J M}\left(\hat{\mathbf{r}}^{\prime}\right),
$$

and we want to find the Fourier transform with respect to $\mathbf{r}$, regarding $\mathbf{r}^{\prime}$ as a parameter. By using Eqs. C.6 and C.7, we find that under Fourier transformation

$$
\frac{\delta\left(r-r^{\prime}\right)}{r r^{\prime}} Y_{J M}^{*}(\hat{\mathbf{r}}) \rightarrow( \pm i)^{J} 4 \pi j_{J}\left(q r^{\prime}\right) Y_{J M}^{*}(\hat{\mathbf{q}})
$$


The transform of the three-dimensional delta function is

$$
\int d \mathbf{r} e^{ \pm i \mathbf{q} \cdot \mathbf{r}} \delta\left(\mathbf{r}-\mathbf{r}^{\prime}\right)=e^{ \pm i \mathbf{q} \cdot \mathbf{r}^{\prime}}
$$

The three-dimensional delta function can be represented as

$$
\delta\left(\mathbf{r}-\mathbf{r}^{\prime}\right)=\frac{1}{(2 \pi)^{3}} \int d \mathbf{q} e^{ \pm i \mathbf{q} \cdot\left(\mathbf{r}-\mathbf{r}^{\prime}\right)} .
$$

We show here a similar relation for the radial delta function. To do this, we write

$$
e^{ \pm i \mathbf{q} \cdot\left(\mathbf{r}-\mathbf{r}^{\prime}\right)}=e^{ \pm i \mathbf{q} \cdot \mathbf{r}} e^{\mp i \mathbf{q} \cdot \mathbf{r}^{\prime}}
$$

and expand both factors on the right in spherical harmonics using Eqs. C.3-C.4. We integrate the resulting expression over $\mathbf{q}$, use the orthogonality relation for the spherical harmonics, and divide by $(2 \pi)^{3}$. This yields

$$
\frac{1}{(2 \pi)^{3}} \int d \mathbf{q} e^{ \pm i \mathbf{q} \cdot\left(\mathbf{r}-\mathbf{r}^{\prime}\right)}=\sum_{J M} \frac{2}{\pi} \int_{0}^{\infty} d q q^{2} j_{J}(q r) j_{J}\left(q r^{\prime}\right) Y_{J M}^{*}(\hat{\mathbf{r}}) Y_{J M}\left(\hat{\mathbf{r}}^{\prime}\right) .
$$

Since the expression on the left is just the three-dimensional delta function, we may compare this equation with Eq. C.49 to find an orthogonality relation for the spherical Bessel functions,

$$
\frac{\delta\left(r-r^{\prime}\right)}{r r^{\prime}}=\frac{2}{\pi} \int_{0}^{\infty} d q q^{2} j_{J}(q r) j_{J}\left(q r^{\prime}\right)
$$

or by interchanging $r$ and $q$ coordinates,

$$
\frac{\delta\left(q-q^{\prime}\right)}{q q^{\prime}}=\frac{2}{\pi} \int_{0}^{\infty} d r r^{2} j_{J}(q r) j_{J}\left(q^{\prime} r\right)
$$




\section{Bibliography}

[1] F. Petrovich, J. A. Carr, and H. McManus, Ann. Rev. Nucl. Part. Sci. 36, 29 (1986).

[2] A. R. Edmonds, Angular Momentum in Quantum Mechanics, 2nd edition (Princeton University Press, Princeton, 1960).

[3] I. J. Thompson, Fresco, Ver. FRXY.3f, unpublished, 2005.

[4] I. J. Thompson, Comp. Phys. Reports 7, 167 (1988).

[5] T. Tamura, Rev. Mod. Phys. 37, 679 (1965).

[6] J. A. Carr, J. J. Kelly, and F. Petrovich, computer code ALLwRLd, unpublished.

[7] P. Ring and P. Schuck, The Nuclear Many-Body Problem (Springer-Verlag, New York, 1981).

[8] M. DuPuis, Ph.D. thesis, Université de Bordeaux I - Talence, 2006.

[9] A. M. Lane and R. G. Thomas, Rev. Mod. Phys. 30, 257 (1958).

[10] W. E. Parker et al., Phys. Rev. C 52, 252 (1995).

[11] A. de Shalit and I. Talmi, Nuclear Shell Theory (Academic Press, New York, 1963).

[12] M. Rotenberg, R. Bivins, N. Metropolis, and J. K. Wooten, the 3-j and 6-j symbols (The Technology Press, MIT, Cambridge, MA, 1959).

[13] A. Bohr and B. Mottelson, Nuclear Structure, Vol. 1 (Benjamin, New York, 1969).

[14] D. M. Brink and G. R. Satchler, Angular Momentum (Clarendon Press, Oxford, 1962).

[15] M. E. Rose, Elementary Theory of Angular Momentum (Wiley, New York, 1957).

[16] F. Petrovich et al., Nucl. Phys. A383, 355 (1982).

[17] F. Petrovich, Nucl. Phys. A251, 143 (1975). 